\title{
DISCRETE APPROXIMATION OF THE MUMFORD-SHAH FUNCTIONAL IN DIMENSION TWO
}

\author{
Antonin Chambolle ${ }^{1}$ and Gianni Dal Maso ${ }^{2}$
}

\begin{abstract}
The Mumford-Shah functional, introduced to study image segmentation problems, is approximated in the sense of $\Gamma$-convergence by a sequence of integral functionals defined on piecewise affine functions.

Résumé. La fonctionnelle de Mumford et Shah, proposée pour l'étude du problème de la segmentation d'images, est approchée au sens de la $\Gamma$-convergence par une suite de fonctionnelles intégrales définies sur des fonctions affines par morceaux.
\end{abstract}

AMS Subject Classification. 26A45, 49J45, 49Q20, 68U10.

Received: May 11, 1998.

\section{INTRODUCTION}

An important role in the variational approach to image segmentation problems (see [21-23]) is played by the Mumford-Shah functional

$$
\int_{\Omega}|\nabla u(x)|^{2} \mathrm{~d} x+\mathcal{H}^{1}\left(S_{u}\right)+\int_{\Omega}|u(x)-g(x)|^{2} \mathrm{~d} x
$$

where $\Omega$ is a bounded open subset of $\mathbb{R}^{2}$ with Lipschitz boundary, $u$ belongs to the space $S B V(\Omega)$ of special functions of bounded variation on $\Omega$ (see Sect. 2), $\nabla u$ is the approximate gradient of $u, S_{u}$ is the set of essential discontinuity points of $u, \mathcal{H}^{1}$ is the one-dimensional Hausdorff measure, and $g$ is a given function in $L^{\infty}(\Omega)$.

The main difficulty in the study of this functional is the presence of the length term $\mathcal{H}^{1}\left(S_{u}\right)$, and many works have been devoted to the problem of approximating this functional by simpler functionals defined on Sobolev spaces.

As pointed out in [10], it is impossible to obtain a variational approximation of (1.1), leading to the convergence of minimum points, by means of local integral functionals of the form

$$
\int_{\Omega} f_{\varepsilon}(\nabla u(x)) \mathrm{d} x+\int_{\Omega}|u(x)-g(x)|^{2} \mathrm{~d} x,
$$

Keywords and phrases. Free discontinuity problems, special bounded variation $(S B V)$ functions, $\Gamma$-convergence, finite elements.

${ }^{1}$ CEREMADE, CNRS UMR 7534, Université de Paris-Dauphine, 75775 Paris Cedex 16, France.

e-mail: chambolle@ceremade.dauphine.fr

2 SISSA, via Beirut 4, 34013 Trieste, Italy. e-mail: dalmaso@sissa.it 
defined in the Sobolev space $H^{1}(\Omega)$. Indeed, if such an approximation existed, (1.1) would be also the variational limit of the sequence of convex functionals

$$
\int_{\Omega} f_{\varepsilon}^{* *}(\nabla u(x)) \mathrm{d} x+\int_{\Omega}|u(x)-g(x)|^{2} \mathrm{~d} x,
$$

where $f_{\varepsilon}^{* *}$ is the convex envelope of $f_{\varepsilon}$ (see, e.g., Prop. 6.1 and Ex. 3.11 in [13]), and would therefore also be convex. This difficulty can be overcome by introducing an auxiliary variable as in the work of Ambrosio and Tortorelli (see [4,5]), while Braides and Dal Maso [10] recently found an approximation of (1.1) using non-local integral functionals.

In this work we consider only the two-dimensional case and present a different solution. We look for approximations of the form (1.2), putting however a restriction on the function space on which it is defined. Our functionals are discrete, in the sense that they are defined on continuous piecewise affine functions that depend, for a fixed $\varepsilon>0$, only on a finite number of parameters. On the other hand, the approach we propose is different from the finite-elements approximation introduced by Bellettini and Coscia [7], that is based on Ambrosio and Tortorelli's functionals. It is also quite different from the finite-differences methods studied in [11], although some similarities exist and will appear clearly in some parts of the proofs.

The new approximation of the Mumford-Shah functional that we propose, based on adaptive finite elements, is studied in this paper from a theoretical point of view. In a forthcoming paper, Bourdin and Chambolle [9] address the problem of its numerical implementation, and show how it can be performed. Their algorithm alternates nonconvex optimization and mesh optimization. The way the optimal mesh is estimated relies a lot on the construction described in Section 4 and in the Appendix, that shows how the triangles need to be oriented in order to approximate well the energy of a given function.

In order to be more precise we need to introduce a few notations and definitions. A triangulation of $\Omega$ is, as usual, a finite family of closed triangles whose union contains $\Omega$ and such that, given any two triangles of this family, their intersection, if not empty, is exactly a vertex or an edge common to both triangles. Given some angle $\theta_{0}$, with $0<\theta_{0} \leq 60^{\circ}$, and a function $\omega(\varepsilon)$, with $\omega(\varepsilon) \geq 6 \varepsilon$ for any $\varepsilon>0$ and $\lim _{\varepsilon \rightarrow 0^{+}} \omega(\varepsilon)=0$, we define, for any $\varepsilon>0, \mathcal{T}_{\varepsilon}(\Omega)=\mathcal{T}_{\varepsilon}\left(\Omega, \omega, \theta_{0}\right)$ as the set of all triangulations of $\Omega$ made of triangles whose edges have length between $\varepsilon$ and $\omega(\varepsilon)$, and whose angles are all greater than or equal to $\theta_{0}$. Then we consider the set $V_{\varepsilon}(\Omega)$ of all continuous functions $u: \Omega \rightarrow \mathbb{R}$ for which there exists $\mathbf{T} \in \mathcal{T}_{\varepsilon}(\Omega)$ such that $u$ is affine on any triangle $T \in \mathbf{T}$. We fix, like in [10], a non-decreasing continuous function $f:[0,+\infty) \rightarrow[0,+\infty)$ such that

$$
\lim _{t \rightarrow 0+} \frac{f(t)}{t}=1 \quad \text { and } \quad \lim _{t \rightarrow+\infty} f(t)=f_{\infty}<+\infty
$$

(the simplest case is the function $f(t)=\min \left\{t, f_{\infty}\right\}$ ), and we set $\mu=f_{\infty} \sin \theta_{0}$. Eventually, we fix some $p \in[1,+\infty)$ and define for every $\varepsilon>0$ the functionals $F_{\varepsilon}: L^{p}(\Omega) \rightarrow[0,+\infty]$ and $F: L^{p}(\Omega) \rightarrow[0,+\infty]$ by

$$
\begin{aligned}
& F_{\varepsilon}(u)= \begin{cases}\frac{1}{\varepsilon} \int_{\Omega} f\left(\varepsilon|\nabla u(x)|^{2}\right) \mathrm{d} x, & \text { if } u \in V_{\varepsilon}(\Omega), \\
+\infty, & \text { if } u \in L^{p}(\Omega) \backslash V_{\varepsilon}(\Omega),\end{cases} \\
& F(u)= \begin{cases}\int_{\Omega}|\nabla u(x)|^{2} \mathrm{~d} x+\mu \mathcal{H}^{1}\left(S_{u}\right), & \text { if } u \in L^{p}(\Omega) \cap G S B V(\Omega), \\
+\infty, & \text { if } u \in L^{p}(\Omega) \backslash G S B V(\Omega),\end{cases}
\end{aligned}
$$

where $G S B V(\Omega)$ is the space of generalized special functions of bounded variation in $\Omega$ and $S_{u}$ is the jump set of $u$ (we refer to the next section for the precise definitions). Note that it is not too difficult to check that, for any $\varepsilon>0, V_{\varepsilon}(\Omega)$ is weakly closed and that the functional $F_{\varepsilon}$ is weakly lower semi-continuous in $L^{p}(\Omega)$.

The main result of the paper is the proof of the $\Gamma$-convergence, as $\varepsilon \rightarrow 0$, of the functionals $F_{\varepsilon}$ to $F$. 
Theorem 1.1. There exists a constant $\Theta$, with $0<\Theta \leq 60^{\circ}$, such that, if $0<\theta_{0}<\Theta$, then the family $\left(F_{\varepsilon}\right)$ $\Gamma$-converges to $F$ in $L^{p}(\Omega)$ as $\varepsilon \rightarrow 0$.

As a consequence of this theorem we will then establish the following result.

Theorem 1.2. Let $g \in L^{\infty}(\Omega), 0<\theta_{0}<\Theta$, and $p>1$. For each $\varepsilon>0$, let $u_{\varepsilon} \in V_{\varepsilon}(\Omega)$ be a minimizer of

$$
F_{\varepsilon}(u)+\int_{\Omega}|u(x)-g(x)|^{p} \mathrm{~d} x .
$$

Then, the family $\left(u_{\varepsilon}\right)_{\varepsilon>0}$ is relatively compact in $L^{p}(\Omega)$ and any limit point $u \in L^{p}(\Omega)$ is a minimizer of

$$
F(u)+\int_{\Omega}|u(x)-g(x)|^{p} \mathrm{~d} x .
$$

Remark 1.3. The optimal value for $\Theta$ is not known, but the construction in the Appendix will show that $\Theta \geq 45^{\circ}-\operatorname{arctg}(1 / 2)>18^{\circ}$.

Remark 1.4. The construction in Section 4 will show that the conclusion of Theorem 1.2 remains valid (including the case $p=1$ ) when the minimizers $u_{\varepsilon}$ are computed under the additional constraint $\left\|u_{\varepsilon}\right\|_{L^{\infty}(\Omega)} \leq\|g\|_{L^{\infty}(\Omega)}$.

Remark 1.5. The proofs in this paper may be easily adapted to the following variant. For any triangle $T$ let $h_{T}$ be the smallest height of the triangle $T$. If $u \in V_{\varepsilon}(\Omega)$ we say that $\mathbf{T} \in \mathcal{T}_{\varepsilon}(\Omega)$ is adapted to $u$ if $\left.u\right|_{T}$ is affine for any $T \in \mathbf{T}$; the set of the triangulations of $\mathcal{T}_{\varepsilon}(\Omega)$ adapted to $u$ is denoted by $\mathcal{T}_{\varepsilon}(u)$. If $\mathbf{T} \in \mathcal{T}_{\varepsilon}(u)$, let $\nabla u_{T}$ be the gradient of $u$ on any $T \in \mathbf{T}$. For any $u \in L^{p}(\Omega)$ we define

$$
\begin{aligned}
G_{\varepsilon}(u) & = \begin{cases}\inf _{\mathbf{T} \in \mathcal{T}_{\varepsilon}(u)} \sum_{T \in \mathbf{T}}|T \cap \Omega| \frac{1}{h_{T}} f\left(h_{T}\left|\nabla u_{T}\right|^{2}\right), & \text { if } u \in V_{\varepsilon}(\Omega), \\
+\infty, & \text { otherwise, }\end{cases} \\
G(u) & = \begin{cases}\int_{\Omega}|\nabla u(x)|^{2} \mathrm{~d} x+f_{\infty} \mathcal{H}^{1}\left(S_{u}\right), & \text { if } u \in L^{p}(\Omega) \cap G S B V(\Omega), \\
+\infty, & \text { if } u \in L^{p}(\Omega) \backslash G S B V(\Omega) .\end{cases}
\end{aligned}
$$

Then, Theorems 1.1 and 1.2 hold with $G_{\varepsilon}$ and $G$ instead of $F_{\varepsilon}$ and $F$, provided $\omega(\varepsilon) \leq c \varepsilon$ for some $c>0$ (another possibility is to replace in the above formulas $h_{T}$ with $\min \left\{h_{T}, c \varepsilon\right\}$, in which case this constraint on $\omega(\varepsilon)$ is no more needed). In this paper we prefer to use $F_{\varepsilon}$, whose meaning is more immediate, but the numerical implementation (see [9]) of $G_{\varepsilon}$ is much simpler, as a better value of the minimum can be reached even when the triangulation is not perfectly optimized along the discontinuity sets. Whereas, with the functional $F_{\varepsilon}$, the optimal triangulation must be made of the smallest possible triangles aligned along the discontinuity, as shown in Section 4.

In the next section we will define precisely the spaces $S B V(\Omega)$ and $G S B V(\Omega)$, and give the definition of the $\Gamma$-convergence. Then the remaining three sections will be devoted to the proof of Theorems 1.1 and 1.2.

\section{Notation AND PRELIMINARIES}

The scalar product of $x, y \in \mathbb{R}^{2}$ is denoted by $\langle x, y\rangle$ and the Euclidean norm by $|x|$. The open ball with centre $x$ and radius $\rho$ is indicated by $B_{\rho}(x)$. Given some open set $A \subset \mathbb{R}^{2}$ and $\rho>0, A_{\rho}$ is the set $\{x \in A: \operatorname{dist}(x, \partial A)>\rho\}$, where $\operatorname{dist}(x, \partial A)$ is the distance from $x$ to $\partial A$. The Lebesgue measure and the one-dimensional Hausdorff measure of a Borel set $B \subset \mathbb{R}^{2}$ are denoted by $|B|$ and $\mathcal{H}^{1}(B)$, respectively. For the general properties of the Hausdorff measure we refer to $[17,18]$. We use standard notation for Lebesgue spaces $L^{p}(\Omega)$ and Sobolev spaces $H^{1}(\Omega)$. 
Special functions of bounded variation. For the general theory of functions of bounded variation we refer to $[17,18,20,25]$; here we just recall some definitions and results we use in the sequel.

Let $A$ be an open subset of $\mathbb{R}^{2}$ and let $u: A \rightarrow[-\infty,+\infty]$ be a measurable function. We say that $t \in$ $[-\infty,+\infty]$ is the approximate limit of $u$ at $x$, and we write $t=\underset{y \rightarrow x}{\operatorname{ap} \lim } u(y)$, if for every neighbourhood $U$ of $t$ we have

$$
\lim _{\rho \rightarrow 0} \frac{1}{\rho^{2}}\left|\left\{y \in B_{\rho}(x) \cap A: u(y) \notin U\right\}\right|=0 .
$$

We define $S_{u}$ as the subset of $A$ where the approximate limit of $u$ does not exist. It turns out that $S_{u}$ is a Borel set and that $\left|S_{u}\right|=0$.

We say that $u \in L^{1}(A)$ is a function of bounded variation if its distributional first derivatives $D_{1} u=\partial u / \partial x_{1}$ and $D_{2} u=\partial u / \partial x_{2}$ are Radon measures with finite total variation in $A$. The space of functions of bounded variation on $A$ is denoted by $B V(A)$. The gradient $D u$ of $u$ is the $\mathbb{R}^{2}$-valued Radon measure $D u=\left(D_{1} u, D_{2} u\right)$.

If $u \in B V(A)$, then $S_{u}$ is countably $\left(\mathcal{H}^{1}, 1\right)$ rectifiable, i.e.,

$$
S_{u}=N \cup \bigcup_{i} K_{i}
$$

where $\mathcal{H}^{1}(N)=0$ and $\left(K_{i}\right)$ is a sequence of compact sets, each one contained in a $C^{1}$ curve $\Gamma_{i}$. Moreover, there exists a Borel function $\nu_{u}: S_{u} \rightarrow S^{1}=\left\{x \in \mathbb{R}^{2}:|x|=1\right\}$ such that the vector $\nu_{u}$ is normal to $S_{u}$, in the sense that, if $S_{u}$ is represented as above, then $\nu_{u}(x)$ is normal to $\Gamma_{i}$ for $\mathcal{H}^{1}$-a.e. $x \in K_{i}$. In particular, for every $u, v \in B V(A)$ it follows that $\nu_{u}(x)= \pm \nu_{v}(x)$ for $\mathcal{H}^{1}$-a.e. $x \in S_{u} \cap S_{v}$.

For every $u \in B V(A)$ we have the Lebesgue decomposition $D u=D^{a} u+D^{s} u$, where $D^{a} u$ is absolutely continuous and $D^{s} u$ is singular with respect to the Lebesgue measure. The density of $D^{a} u$ with respect to the Lebesgue measure is denoted by $\nabla u$. It turns out that for almost every $x \in A$ the vector $\nabla u(x)$ is the approximate gradient of $u$, i.e.,

$$
\operatorname{ap}_{y \rightarrow x} \frac{u(y)-u(x)-\langle\nabla u(x), y-x\rangle}{|y-x|}=0 .
$$

We say that a function $u \in B V(A)$ is a special function of bounded variation if the singular part of $D u$ is concentrated on $S_{u}$, i.e., $\left|D^{s} u\right|\left(A \backslash S_{u}\right)=0$. The space of special functions of bounded variation is denoted by $S B V(A)$. We consider also the larger space $G S B V(A)$, which is composed of all measurable functions $u: A \rightarrow[-\infty,+\infty]$ whose truncations $u^{k}=(u \wedge k) \vee(-k)$ belong to $S B V\left(A^{\prime}\right)$ for every $k>0$ and for every open set $A^{\prime} \subset \subset A$, i.e., with $\bar{A}^{\prime}$ compact and contained in $A$.

Every $u \in G S B V(A) \cap L^{1}(A)$ has an approximate gradient $\nabla u(x)$ for almost every $x \in A$ and

$$
\begin{array}{rlrl}
\left|\nabla u^{k}(x)\right| & \leq|\nabla u(x)| & \text { a.e. in } A, \\
\nabla u^{k}(x) & \rightarrow \nabla u(x) \quad \text { a.e. in } A \text { as } k \rightarrow \infty, \\
S_{u^{k}} & \subset S_{u}, & & \\
\mathcal{H}^{1}\left(S_{u^{k}}\right) & \rightarrow \mathcal{H}^{1}\left(S_{u}\right) & & \text { as } k \rightarrow \infty .
\end{array}
$$

The spaces $S B V(A)$ and $G S B V(A)$ have been introduced by De Giorgi and Ambrosio in [14], and have been studied in $[1,3]$.

The following compactness and lower semi-continuity result is proved in [1] (see also [3]).

Theorem 2.1. Let $A$ be a bounded open subset of $\mathbb{R}^{2}$ and let $\left(u_{j}\right)$ be a sequence in $G S B V(A)$. Suppose that there exist two constants $p$ and $C$, with $1 \leq p \leq+\infty$ and $0 \leq C<+\infty$, such that

$$
\int_{A}\left|\nabla u_{j}\right|^{2} \mathrm{~d} x+\mathcal{H}^{1}\left(S_{u_{j}}\right)+\left\|u_{j}\right\|_{L^{p}(A)} \leq C
$$


for every $j$. Then there exist a subsequence (not relabelled) and a function $u \in G S B V(A)$ such that

$$
\begin{array}{rlrl}
u_{j}(x) & \rightarrow u(x) & & \text { a.e. in } A, \\
\nabla u_{j} & \rightarrow \nabla u & \text { weakly in } L^{2}\left(A ; \mathbb{R}^{2}\right), \\
\mathcal{H}^{1}\left(S_{u}\right) & \leq \liminf _{j \rightarrow \infty} \mathcal{H}^{1}\left(S_{u_{j}}\right) & &
\end{array}
$$

Moreover

$$
\int_{S_{u}}\left|\left\langle\nu_{u}(x), \xi\right\rangle\right| \mathrm{d} \mathcal{H}^{1}(x) \leq \liminf _{j \rightarrow \infty} \int_{S_{u_{j}}}\left|\left\langle\nu_{u_{j}}(x), \xi\right\rangle\right| \mathrm{d} \mathcal{H}^{1}(x)
$$

for every $\xi \in S^{1}$.

$\Gamma$-convergence. Let $X$ be a metric space and let $\left(F_{j}\right)$ be a sequence of functions defined on $X$ with values in $[-\infty,+\infty]$. For every $u \in X$ we set (see [15])

$$
\begin{aligned}
F^{\prime}(u) & =\Gamma-\liminf _{j \rightarrow \infty} F_{j}(u)=\inf \left\{\liminf _{j \rightarrow \infty} F_{j}\left(u_{j}\right): u_{j} \rightarrow u\right\}, \\
F^{\prime \prime}(u) & =\Gamma-\limsup _{j \rightarrow \infty} F_{j}(u)=\inf \left\{\limsup _{j \rightarrow \infty} F_{j}\left(u_{j}\right): u_{j} \rightarrow u\right\} .
\end{aligned}
$$

Let $F$ be a function defined on $X$ with values in $[-\infty,+\infty]$. We say that $\left(F_{j}\right) \Gamma$-converges to $F$ in $X$ if $F^{\prime}=F^{\prime \prime}=F$ in $X$. This means that for every $u \in X$ the following two conditions are satisfied:

(a) for every sequence $\left(u_{j}\right)$ converging to $u$ we have $F(u) \leq \liminf _{j \rightarrow \infty} F_{j}\left(u_{j}\right)$;

(b) there exists a sequence $\left(u_{j}\right)$ converging to $u$ such that $F(u)=\lim _{j \rightarrow \infty} F_{j}\left(u_{j}\right)$.

It turns out that the functions $F^{\prime}$ and $F^{\prime \prime}$ are lower semi-continuous on $X$. For the main properties of $\Gamma$-convergence we refer to $[6,13,15]$.

Let $\left(F_{\varepsilon}\right)_{\varepsilon>0}$ be a family of functionals defined on $X$ with values in $[-\infty,+\infty]$ and let $F: X \rightarrow[-\infty,+\infty]$. We say that $\left(F_{\varepsilon}\right) \Gamma$-converges to $F$ in $X$ as $\varepsilon \rightarrow 0$ if $\left(F_{\varepsilon_{j}}\right) \Gamma$-converges to $F$ in $X$ for every sequence $\left(\varepsilon_{j}\right)$ of positive real numbers converging to 0 as $j \rightarrow \infty$.

\section{Estimate of the $\Gamma$-Limit From Below}

We now return to the problem introduced in Section 1. In order to prove Theorem 1.1, we will first show that the $\Gamma$-liminf of the family $\left(F_{\varepsilon}\right)_{\varepsilon>0}$ is greater than or equal to $F$. Then, in the next section, we will show the opposite inequality for the $\Gamma$-limsup.

For every open set $A \subset \Omega$ and for every $\varepsilon>0$ we consider the following "localization" of $F_{\varepsilon}$ :

$$
F_{\varepsilon}(u, A)= \begin{cases}\frac{1}{\varepsilon} \int_{A} f\left(\varepsilon|\nabla u(x)|^{2}\right) \mathrm{d} x, & \text { if } u \in V_{\varepsilon}(\Omega), \\ +\infty, & \text { if } u \in L^{p}(\Omega) \backslash V_{\varepsilon}(\Omega) .\end{cases}
$$

We choose an arbitrary sequence of positive numbers $\left(\varepsilon_{j}\right)$ converging to 0 as $j \rightarrow \infty$, and we set

$$
F^{\prime}(u, A)=\Gamma-\liminf _{j \rightarrow \infty} F_{\varepsilon_{j}}(u, A)
$$


for every $u \in L^{p}(\Omega)$ and every open set $A \subset \Omega$. We wish to prove that

$$
F^{\prime}(u, \Omega) \geq \int_{\Omega}|\nabla u(x)|^{2} \mathrm{~d} x+\mu \mathcal{H}^{1}\left(S_{u}\right)
$$

for any $u \in L^{p}(\Omega) \cap G S B V(\Omega)$. In order to do so we use the same localization technique as in [10]. The first step will be to establish the following result.

Proposition 3.1. Let $u \in L^{p}(\Omega)$ and let $A$ be an open subset of $\Omega$ such that $F^{\prime}(u, A)<+\infty$. Then $u \in$ $G S B V(A)$ and

$$
\begin{aligned}
\mathcal{H}^{1}\left(S_{u} \cap A\right) & <+\infty \\
F^{\prime}(u, A) & \geq \int_{A}|\nabla u(x)|^{2} \mathrm{~d} x, \\
F^{\prime}(u, A) & \geq \mu \int_{S_{u} \cap A}\left|\left\langle\nu_{u}(x), \xi\right\rangle\right| \mathrm{d} \mathcal{H}^{1}(x)
\end{aligned}
$$

for every $\xi \in S^{1}$.

We point out that given $u \in L^{p}(\Omega)$, the set functions $A \mapsto F_{\varepsilon}(u, A)$ are increasing and super-additive, and thus the set function $A \mapsto F^{\prime}(u, A)$ is also increasing and super-additive:

(i) $F^{\prime}\left(u, A_{1}\right) \leq F^{\prime}\left(u, A_{2}\right)$ whenever $A_{1} \subset A_{2} \subset \Omega$;

(ii) $F^{\prime}\left(u, A_{1} \cup A_{2}\right) \geq F^{\prime}\left(u, A_{1}\right)+F^{\prime}\left(u, A_{2}\right)$ whenever $A_{1} \cap A_{2}=\emptyset$.

The next corollary is a consequence of this remark and of the previous proposition.

Corollary 3.2. Let $u \in L^{p}(\Omega)$ and let $A$ be an open subset of $\Omega$ such that $F^{\prime}(u, A)<+\infty$. Then $u \in G S B V(A)$ and

$$
F^{\prime}(u, A) \geq \int_{A}|\nabla u(x)|^{2} \mathrm{~d} x+\mu \mathcal{H}^{1}\left(S_{u} \cap A\right) .
$$

We will not give the proof of this corollary, since it is identical to the proof of the similar Proposition 6.5 in [10] (see also Lem. 4.1(iii) and Prop. 4.3 in [1], or Lem. 2.4 in [8]).

Our task is now to prove Proposition 3.1. This proof will be split into two parts: first we will show $(3.2,3.3)$, that will be deduced quite easily from next Proposition 3.3; later on, as a consequence of Proposition 3.4, we will establish inequality (3.4).

Proposition 3.3. For every $\delta>0$ we can determine two constants $c_{\delta}, c_{\delta}^{\prime}>0$ such that, for every $\varepsilon>0$, for every $u \in V_{\varepsilon}(\Omega)$, and for every open subset $A$ of $\Omega$, there exists $v \in S B V(\Omega)$ with

$$
\begin{aligned}
(1-\delta) \int_{A_{\omega(\varepsilon)}}|\nabla v(x)|^{2} \mathrm{~d} x+c_{\delta} \mathcal{H}^{1}\left(S_{v} \cap A_{\omega(\varepsilon)}\right) & \leq F_{\varepsilon}(u, A), \\
|\{x \in \Omega: v(x) \neq u(x)\}| & \leq c_{\delta}^{\prime} \varepsilon F_{\varepsilon}(u, A) .
\end{aligned}
$$

Proof. Let us choose $\delta \in(0,1)$. From the properties of the function $f$, there exists a constant $c$, with $0<c \leq f_{\infty}$, such that

$$
f(t) \geq \min \{(1-\delta) t, c\}
$$

for all $t \geq 0$. Let $u \in V_{\varepsilon}(\Omega)$ and assume $F_{\varepsilon}(u, A)<+\infty$ (otherwise the result is obvious), and let $\mathbf{T} \in \mathcal{T}_{\varepsilon}(\Omega)$ be a triangulation such that $\left.u\right|_{T}$ is affine for any triangle $T \in \mathbf{T}$. For each of these triangles $T$ let $\nabla u_{T}$ be 
the (constant) gradient of $u$ on $T$. Observe that, letting $\mathbf{T}_{A}=\{T \in \mathbf{T}: T \subset A\}$, we have

$$
\begin{aligned}
F_{\varepsilon}(u, A) & =\sum_{T \in \mathbf{T}}|T \cap A| \frac{1}{\varepsilon} f\left(\varepsilon\left|\nabla u_{T}\right|^{2}\right) \\
& \geq \sum_{T \in \mathbf{T}_{A}}|T| \frac{1}{\varepsilon} \min \left\{\varepsilon(1-\delta)\left|\nabla u_{T}\right|^{2}, c\right\} \\
& =\sum_{T \in \mathbf{T}_{A}}|T| \min \left\{(1-\delta)\left|\nabla u_{T}\right|^{2}, \frac{c}{\varepsilon}\right\} .
\end{aligned}
$$

Let $\mathbf{T}_{1}=\left\{T \in \mathbf{T}_{A}:(1-\delta)\left|\nabla u_{T}\right|^{2}>c / \varepsilon\right\}$ and $\mathbf{T}_{2}=\mathbf{T}_{A} \backslash \mathbf{T}_{1}$, and define $v$ in the following way:

$$
v= \begin{cases}0, & \text { on every } T \in \mathbf{T}_{1}, \\ u, & \text { on every } T \in \mathbf{T} \backslash \mathbf{T}_{1} .\end{cases}
$$

Clearly, $v \in S B V(\Omega)$ and each point of $S_{v}$ belongs to the boundary $\partial T$ of some triangle $T \in \mathbf{T}_{1}$; moreover,

$$
|\{x \in \Omega: v(x) \neq u(x)\}| \leq \sum_{T \in \mathbf{T}_{1}}|T| \leq \frac{\varepsilon}{c} F_{\varepsilon}(u, A),
$$

where the last inequality follows from (3.7), so that (3.6) holds with $c_{\delta}^{\prime}=1 / c$.

By assumption the edges of the triangles of $\mathbf{T}$ have length greater than or equal to $\varepsilon$ and their angles are all greater than or equal to $\theta_{0}$, so that the heights of such triangles must be greater than or equal to $\varepsilon \sin \theta_{0}$. Therefore, if $T \in \mathbf{T}$,

so that

$$
|T| \geq \frac{1}{2} \cdot \varepsilon \sin \theta_{0} \cdot \frac{\mathcal{H}^{1}(\partial T)}{3},
$$

$$
\frac{c}{\varepsilon}|T| \geq c_{\delta} \mathcal{H}^{1}(\partial T)
$$

if $c_{\delta}=\left(c \sin \theta_{0}\right) / 6$. Moreover, since the edges of each triangle $T \in \mathbf{T}$ have length less than or equal to $\omega(\varepsilon)$, we also have that $T \cap A_{\omega(\varepsilon)} \neq \emptyset$ implies $T \subset A$, thus

$$
\begin{aligned}
(1-\delta) \int_{A_{\omega(\varepsilon)}}|\nabla v(x)|^{2} \mathrm{~d} x+c_{\delta} \mathcal{H}^{1}\left(S_{v} \cap A_{\omega(\varepsilon)}\right) & \leq(1-\delta) \sum_{T \in \mathbf{T}_{2}}\left|T \cap A_{\omega(\varepsilon)}\right|\left|\nabla u_{T}\right|^{2}+c_{\delta} \sum_{T \in \mathbf{T}_{1}} \mathcal{H}^{1}\left(\partial T \cap A_{\omega(\varepsilon)}\right) \\
& \leq \sum_{T \in \mathbf{T}_{2}}|T|(1-\delta)\left|\nabla u_{T}\right|^{2}+\sum_{T \in \mathbf{T}_{1}} \frac{c}{\varepsilon}|T| \leq F_{\varepsilon}(u, A)
\end{aligned}
$$

by (3.7), so that (3.5) holds and the proof Proposition (3.3) is achieved.

Proof of (3.2, 3.3). Let now $u \in L^{p}(\Omega)$ and assume that $F^{\prime}(u, A)<+\infty$ for some open set $A \subset \Omega$. We consider an arbitrary sequence $\left(u_{j}\right)$ such that $u_{j} \rightarrow u$ in $L^{p}(\Omega)$ and $\liminf _{j} F_{\varepsilon_{j}}\left(u_{j}, A\right)<+\infty$. Without loss of generality we may assume (possibly extracting a subsequence) that $\sup _{j} F_{\varepsilon_{j}}\left(u_{j}, A\right)=c<+\infty$. Let us fix $\delta \in(0,1)$. For any $j$, Proposition 3.3 provides a function $v_{j}$, that satisfies $(3.5,3.6)$ (with $u=u_{j}, v=v_{j}$, and $\varepsilon=\varepsilon_{j}$ ). Choose an arbitrary truncation level $k>0$ and define

$$
v_{j}^{k}=\left(-k \vee v_{j}\right) \wedge k \quad \text { and } \quad u_{j}^{k}=\left(-k \vee u_{j}\right) \wedge k .
$$

Clearly, $u_{j}^{k} \rightarrow(-k \vee u) \wedge k=u^{k}$ in $L^{p}(\Omega)$ (thus in $\left.L^{1}(\Omega)\right)$ as $j \rightarrow \infty$, moreover by (3.6)

$$
\int_{\Omega}\left|v_{j}^{k}(x)-u_{j}^{k}(x)\right| \mathrm{d} x \leq 2 k c_{\delta}^{\prime} \varepsilon_{j} F_{\varepsilon_{j}}\left(u_{j}, A\right) \leq 2 k c_{\delta}^{\prime} c \varepsilon_{j}
$$


so that $v_{j}^{k}$ also converges to $u^{k}$ in $L^{1}(\Omega)$.

Let $\eta>0$. By (3.5)

$$
(1-\delta) \int_{A_{\eta}}\left|\nabla v_{j}^{k}(x)\right|^{2} \mathrm{~d} x+c_{\delta} \mathcal{H}^{1}\left(S_{v_{j}^{k}} \cap A_{\eta}\right) \leq F_{\varepsilon_{j}}\left(u_{j}, A\right) \leq c
$$

as soon as $A_{\eta} \subset A_{\omega\left(\varepsilon_{j}\right)}$, that is to say, when $j$ is large enough, and by Ambrosio's Theorem 2.1 we deduce that $u^{k} \in S B V\left(A_{\eta}\right)$ and

$$
(1-\delta) \int_{A_{\eta}}\left|\nabla u^{k}(x)\right|^{2} \mathrm{~d} x+c_{\delta} \mathcal{H}^{1}\left(S_{u^{k}} \cap A_{\eta}\right) \leq \liminf _{j \rightarrow \infty} F_{\varepsilon_{j}}\left(u_{j}, A\right) .
$$

As $\eta$ and the sequence $\left(u_{j}\right)$ were arbitrary, we conclude that $u^{k} \in S B V(A)$ and

$$
(1-\delta) \int_{A}\left|\nabla u^{k}(x)\right|^{2} \mathrm{~d} x+c_{\delta} \mathcal{H}^{1}\left(S_{u^{k}} \cap A\right) \leq F^{\prime}(u, A) .
$$

Sending $k$ to infinity and invoking (2.1), we find that the same inequality holds replacing $u^{k}$ with $u$. Therefore (3.2) holds, and letting $\delta$ go to zero we also get (3.3).

We now concentrate on the length term, and will prove the next proposition, from which (3.4) will follow.

Proposition 3.4. For every $\delta>0$ we can determine three constants $c_{\delta}, c_{\delta}^{\prime}, c_{\delta}^{\prime \prime}>0$ such that, for every $\varepsilon>0$, for every $u \in V_{\varepsilon}(\Omega)$, for every $\xi \in S^{1}$, and for every open subset $A$ of $\Omega$, there exists $v \in S B V(\Omega)$ with

$$
\begin{aligned}
(1-\delta) \mu \int_{S_{v} \cap A_{\omega(\varepsilon)}}\left|\left\langle\nu_{v}(x), \xi\right\rangle\right| \mathrm{d} \mathcal{H}^{1}(x) & \leq F_{\varepsilon}(u, A), \\
c_{\delta} \int_{A_{\omega(\varepsilon)}}|\nabla v(x)|^{2} \mathrm{~d} x+c_{\delta}^{\prime} \mathcal{H}^{1}\left(S_{v} \cap A_{\omega(\varepsilon)}\right) & \leq F_{\varepsilon}(u, A), \\
|\{x \in \Omega: v(x) \neq u(x)\}| & \leq c_{\delta}^{\prime \prime} \varepsilon F_{\varepsilon}(u, A) .
\end{aligned}
$$

Proof. The function $v$ will be constructed from $u$ more or less like in the proof of Proposition 3.3, but this time we will need to describe more precisely the way we "cut" the triangles with large gradient. Here, again, we choose $\delta \in(0,1)$, and from the properties of the function $f$ we find a constant $c$, with $0<c<1$, such that

$$
f(t) \geq \min \left\{c t,(1-\delta) f_{\infty}\right\}
$$

for all $t \geq 0$. We consider some function $u \in V_{\varepsilon}(\Omega)$ with $F_{\varepsilon}(u, A)<+\infty$, a triangulation $\mathbf{T} \in \mathcal{T}_{\varepsilon}(\Omega)$ such that $u$ is affine on any triangle $T \in \mathbf{T}$, and we still write $\nabla u_{T}$ for the gradient of $u$ on $T$. We also set $\mathbf{T}_{A}=\{T \in \mathbf{T}: T \subset A\}$.

Let $\sigma=\sqrt{(1-\delta) f_{\infty} / c}$; we now classify the triangles $T \in \mathbf{T}_{A}$ into four categories, depending on the slope of the function $u$ along the edges of $T$ :

- $\mathbf{T}_{0}$ is the set of triangles $T \in \mathbf{T}_{A}$ such that, along all three edges of $T$, the slope of $u$ is less than or equal to $\sigma / \sqrt{\varepsilon}$,

- for each $i=1,2,3, \mathbf{T}_{i}$ is the set of the triangles $T \in \mathbf{T}_{A}$ such that the slope of $u$ is greater than $\sigma / \sqrt{\varepsilon}$ along exactly $i$ edges of $T$. 
We make the following remarks.

Remark 3.5. If $T$ is a triangle with all three angles greater than $\theta_{0}$ and if $w: T \rightarrow \mathbb{R}$ is an affine function such that the slopes of $w$ along at least two of the edges of $T$ are smaller than some constant $K$, then

$$
|\nabla w| \leq K \frac{\sqrt{5}}{\sin \theta_{0}}
$$

Indeed, we may always assume that one of these two edges is along the horizontal $x_{1}$-axis of the plane while the other is along some vector $(\cos \theta, \sin \theta)$, with $\theta_{0} \leq \theta \leq \pi-2 \theta_{0}$, so that $\sin \theta \geq \sin \theta_{0}>0$. By assumption, $\left|D_{1} w\right| \leq K$ and $\left|D_{1} w \cos \theta+D_{2} w \sin \theta\right| \leq K$. Thus, $\left|D_{2} w\right| \sin \theta \leq K+\left|D_{1} w\right| \leq 2 K$ and

$$
|\nabla w| \leq\left(1+\frac{4}{\sin ^{2} \theta}\right)^{\frac{1}{2}} K
$$

hence (3.11) holds. In particular, we notice that if $T \in \mathbf{T}_{0} \cup \mathbf{T}_{1}$, then

$$
\left|\nabla u_{T}\right| \leq \frac{\sigma}{\sin \theta_{0}} \sqrt{\frac{5}{\varepsilon}}
$$

Remark 3.6. If $c \varepsilon\left|\nabla u_{T}\right|^{2} \leq(1-\delta) f_{\infty}$ for some $T \in \mathbf{T}_{A}$, so that $\left|\nabla u_{T}\right| \leq \sigma / \sqrt{\varepsilon}$, then clearly $T \in \mathbf{T}_{0}$, since the slope along any edge is less than or equal to the norm of the gradient.

Proof of Proposition (3.4). As in (3.7) we can write

$$
\begin{aligned}
F_{\varepsilon}(u, A) & \geq \sum_{T \in \mathbf{T}_{A}} \frac{|T|}{\varepsilon} \min \left\{c \varepsilon\left|\nabla u_{T}\right|^{2},(1-\delta) f_{\infty}\right\} \\
& =c \sum_{T \in \mathbf{T}_{0}}|T| \min \left\{\left|\nabla u_{T}\right|^{2}, \frac{\sigma^{2}}{\varepsilon}\right\}+(1-\delta) f_{\infty} \sum_{T \in \mathbf{T}_{A} \backslash \mathbf{T}_{0}} \frac{|T|}{\varepsilon},
\end{aligned}
$$

where the last equality follows from Remark 3.6. Combining this with (3.12), we find

$$
F_{\varepsilon}(u, A) \geq c^{\prime} \sum_{T \in \mathbf{T}_{0}}|T|\left|\nabla u_{T}\right|^{2}+(1-\delta) \mu \sum_{T \in \mathbf{T}_{A} \backslash \mathbf{T}_{0}} \frac{|T|}{\varepsilon \sin \theta_{0}}
$$

with $c^{\prime}=\left(c \sin ^{2} \theta_{0}\right) / 5$. We will construct the function $v$ by modifying $u$ in each triangle of $\mathbf{T}_{A} \backslash \mathbf{T}_{0}=\mathbf{T}_{1} \cup \mathbf{T}_{2} \cup \mathbf{T}_{3}$. In all the other triangles $T \in \mathbf{T}_{0} \cup\left(\mathbf{T} \backslash \mathbf{T}_{A}\right)$ we first set $\left.v\right|_{T}=\left.u\right|_{T}$, so that (3.10) holds exactly in the same way as (3.6) in Proposition 3.3, with $c_{\delta}^{\prime \prime}=1 /(1-\delta) f_{\infty}$. Then, in order to obtain also $(3.8,3.9)$, we need to define the new function $v$ in each triangle $T \in \mathbf{T}_{1} \cup \mathbf{T}_{2} \cup \mathbf{T}_{3}$ in a way that for any such $T$,

$$
\frac{|T|}{\varepsilon \sin \theta_{0}} \geq \int_{S_{v} \cap A_{\omega(\varepsilon)} \cap T}\left|\left\langle\nu_{v}(x), \xi\right\rangle\right| \mathrm{d} \mathcal{H}^{1}(x),
$$

and

$$
(1-\delta) \mu \frac{|T|}{\varepsilon \sin \theta_{0}} \geq c_{\delta} \int_{A_{\omega(\varepsilon) \cap T}}|\nabla v(x)|^{2} \mathrm{~d} x+c_{\delta}^{\prime} \mathcal{H}^{1}\left(S_{v} \cap A_{\omega(\varepsilon)} \cap T\right)
$$

for some suitable constants $c_{\delta}>0$ and $c_{\delta}^{\prime}>0$. 
We now can detail how $v$ is constructed in each one of the three cases $T \in \mathbf{T}_{1}, T \in \mathbf{T}_{2}$, and $T \in \mathbf{T}_{3}$. The idea will be the following: on the edges of the triangles of $\mathbf{T}_{A}$ along which the slope of $u$ is low (i.e., less than or equal to $\sigma(\sqrt{\varepsilon}), v$ will be equal to $u$, whereas on each edge along which the slope is high (i.e., greater than $\sigma / \sqrt{\varepsilon}), v$ will be piecewise constant, assuming the two values of $u$ at the vertices of the edge and "jumping" in the middle.

Case $T \in \mathbf{T}_{2}$. Call $x_{1}, x_{2}, x_{3}$ the vertices of $T$ and assume that the two edges on which the slope of $u$ is larger than $\sigma / \sqrt{\varepsilon}$ are $\left[x_{1}, x_{2}\right]$ and $\left[x_{2}, x_{3}\right]$, i.e.,

$$
\frac{\left|u\left(x_{2}\right)-u\left(x_{1}\right)\right|}{\left|x_{2}-x_{1}\right|}>\frac{\sigma}{\sqrt{\varepsilon}} \quad \text { and } \quad \frac{\left|u\left(x_{3}\right)-u\left(x_{2}\right)\right|}{\left|x_{3}-x_{2}\right|}>\frac{\sigma}{\sqrt{\varepsilon}}
$$

while

$$
\frac{\left|u\left(x_{3}\right)-u\left(x_{1}\right)\right|}{\left|x_{3}-x_{1}\right|} \leq \frac{\sigma}{\sqrt{\varepsilon}} .
$$

Call then $x_{12}$ the middle point of $\left[x_{1}, x_{2}\right]$ and $x_{23}$ the middle point of $\left[x_{2}, x_{3}\right]$. We "cut" the triangle along the segment $\left[x_{12}, x_{23}\right]$ and define $v(x)$ on each side of this segment. On the "small" triangle $\left[x_{12}, x_{23}, x_{2}\right]$, we set $v(x)=u\left(x_{2}\right)$, and on the quadrangle $\left[x_{1}, x_{2}, x_{23}, x_{12}\right]$ we choose a suitable continuous function $v$ such that

- $v$ is constant, equal to $u\left(x_{1}\right)$ (resp., $u\left(x_{3}\right)$ ) on $\left[x_{1}, x_{12}\right]$ (resp., $\left[x_{3}, x_{23}\right]$ );

- $v$ is affine, on $\left[x_{1}, x_{3}\right]$ and $\left[x_{12}, x_{23}\right]$.

A good choice is to take $v$ affine on each triangle $\left[x_{1}, x_{23}, x_{12}\right]$ and $\left[x_{1}, x_{3}, x_{23}\right]$, with the above mentioned boundary conditions. In this case it is simple to estimate the norm of the gradient of $v$, since the slope of $v$ is zero on $\left[x_{1}, x_{12}\right]$ and $\left[x_{3}, x_{23}\right]$ and less than $\sigma / \sqrt{\varepsilon}$ (resp., $2 \sigma / \sqrt{\varepsilon}$ ) on $\left[x_{1}, x_{3}\right]$ (resp., $\left[x_{12}, x_{23}\right]$ ). Adapting Remark 3.5, we get

$$
\sup _{x \in T}|\nabla v(x)| \leq \frac{2 \sigma}{\sin \theta_{0}} \sqrt{\frac{5}{\varepsilon}}
$$

Actually, using the fact that the slope is zero on $\left[x_{1}, x_{12}\right]$ and $\left[x_{3}, x_{23}\right]$, we could estimate better the gradient in this particular case and show that it is bounded by $2 \sigma /\left(\sin \theta_{0} \sqrt{\varepsilon}\right)$.

Since the area $|T|$ is equal to the length of $\left[x_{12}, x_{23}\right]$ multiplied by the corresponding height, which is greater than $\varepsilon \sin \theta_{0}$, the length of the cut we have made satisfies

$$
\mathcal{H}^{1}\left(\left[x_{12}, x_{23}\right]\right) \leq \frac{|T|}{\varepsilon \sin \theta_{0}},
$$

while

$$
\int_{T}|\nabla v(x)|^{2} \mathrm{~d} x \leq|T| \frac{20 \sigma^{2}}{\varepsilon \sin ^{2} \theta_{0}}=\frac{20}{c \sin ^{2} \theta_{0}}(1-\delta) \mu \frac{|T|}{\varepsilon \sin \theta_{0}} .
$$

Case $T \in \mathbf{T}_{3}$. This time the slope of $u$ is above the threshold $\sigma / \sqrt{\varepsilon}$ along all three edges of $T$. We use the same notation as in the previous case, assuming moreover that $x_{1}, x_{2}$, and $x_{3}$ are ordered in the sense that $\left\langle x_{1}, \xi^{\perp}\right\rangle \leq\left\langle x_{2}, \xi^{\perp}\right\rangle \leq\left\langle x_{3}, \xi^{\perp}\right\rangle$, where $\xi^{\perp}$ is one of the two vectors in $S^{1}$ perpendicular to $\xi$. We call $x_{13}$ the middle of $\left[x_{1}, x_{3}\right]$, notice that $x_{12}, x_{13}$, and $x_{23}$ are also ordered:

$$
\left\langle x_{12}, \xi^{\perp}\right\rangle \leq\left\langle x_{13}, \xi^{\perp}\right\rangle \leq\left\langle x_{23}, \xi^{\perp}\right\rangle .
$$


We cut the triangle $T$ along $\left[x_{12}, x_{13}\right] \cup\left[x_{13}, x_{23}\right]$, and choose $v$ to be piecewise constant in $T$ :

- $v=u\left(x_{1}\right)$ in $\left[x_{1}, x_{12}, x_{13}\right]$,

- $v=u\left(x_{2}\right)$ in $\left[x_{12}, x_{13}, x_{23}, x_{2}\right]$,

- $v=u\left(x_{3}\right)$ in $\left[x_{13}, x_{23}, x_{3}\right]$.

Here $\nabla v=0$, while the length of the cut satisfies

$$
\mathcal{H}^{1}\left(\left[x_{12}, x_{13}\right]\right)+\mathcal{H}^{1}\left(\left[x_{13}, x_{23}\right]\right) \leq 2 \frac{|T|}{\varepsilon \sin \theta_{0}}
$$

(see the proof of (3.17)). Moreover, if we denote the vectors $x_{13}-x_{12}, x_{23}-x_{12}$, and $x_{23}-x_{13}$ by $s_{1}$, $s_{2}$, and $s_{3}$, respectively, we have by $(3.19)$,

$$
\begin{aligned}
\int_{\left[x_{12}, x_{13}\right] \cup\left[x_{13}, x_{23}\right]}\left|\left\langle\nu_{v}(x), \xi\right\rangle\right| \mathrm{d} \mathcal{H}^{1}(x) & =\left\langle s_{1}, \xi^{\perp}\right\rangle+\left\langle s_{3}, \xi^{\perp}\right\rangle \\
& =\left\langle s_{2}, \xi^{\perp}\right\rangle \leq\left|s_{2}\right|=\mathcal{H}^{1}\left(\left[x_{12}, x_{23}\right]\right) \leq \frac{|T|}{\varepsilon \sin \theta_{0}} .
\end{aligned}
$$

Case $T \in \mathbf{T}_{1}$. In this last case, we cut the triangle as in the previous case so that we will also rely on estimates $(3.20,3.21)$ for the length terms. The difference is that we can not define $v$ to be constant in all three connected components of $T \backslash\left(\left[x_{12}, x_{13}\right] \cup\left[x_{13}, x_{23}\right]\right)$, as this time we need it to be affine (and equal to $u$ ) along the two edges on which the slope of $u$ is below $\sigma / \sqrt{\varepsilon}$. The solution is to take $v$ affine in each connected component of $T \backslash\left(\left[x_{12}, x_{13}\right] \cup\left[x_{13}, x_{23}\right]\right)$, with the correct boundary conditions, namely, $v=u$ on the two edges where the slope is below the threshold, and, if $\left[x_{i}, x_{j}\right](1 \leq i<j \leq 3)$ is the third edge, $v=u\left(x_{i}\right)$ on $\left[x_{i}, x_{i j}\right.$ [ and $v=u\left(x_{j}\right)$ on $\left.] x_{i j}, x_{j}\right]$. On each connected component, $v$ is the restriction of some affine function defined on $T$ that has a slope less than $\sigma / \sqrt{\varepsilon}$ on at least two edges of the triangle. Thus, by Remark 3.5, inequality (3.16) and its consequence (3.18) hold also in this case.

In this way, we have constructed a function $v \in S B V(\Omega)$ such that $S_{v}$ is made of the cuts we introduced in the three above mentioned cases, to which me must add the boundary of the union $B$ of all triangles $T \in \mathbf{T}_{A}$ (to be more precise some subset of this boundary). But, by assumption, $A_{\omega(\varepsilon)} \subset \operatorname{int} B$, so that $A_{\omega(\varepsilon)} \cap \partial B=\emptyset$. From $(3.17,3.21)$ we deduce $(3.14)$, while $(3.15)$ follows from $(3.17,3.20,3.18)$, if we choose for instance $c_{\delta}=c\left(\sin ^{2} \theta_{0}\right) / 40$ and $c_{\delta}^{\prime}=(1-\delta) \mu / 4$. Then, we deduce (3.8) from (3.14), while (3.9) is a consequence of $(3.13$, 3.15). The proof of Proposition 3.4 is complete.

Proof of (3.4). Inequality (3.4) is deduced from Proposition 3.4 exactly in the same way as (3.2, 3.3) follow from Proposition 3.3, and we will not repeat the proof here. Just notice that, this time, the last semi-continuity inequality in Theorem 2.1 has to be used, as well as the fact that for any truncation level $k>0$ and any $u \in G S B V(\Omega)$ we have $S_{u^{k}} \subset S_{u}$ and thus $\nu_{u^{k}}(x)=\nu_{u}(x)$ for $\mathcal{H}^{1}$-almost all $x \in S_{u^{k}}$.

The proof of (3.4) achieves the demonstration of Proposition 3.1.

Remark 3.7. Note that in this section we have assumed only that $0<\theta_{0} \leq 60^{\circ}$. The maximum angle $\Theta$ will appear only in the next section, where we find an upper bound for the $\Gamma$-limit of $F_{\varepsilon}$.

\section{Estimate of the $\Gamma$-Limit from ABove}

As in the previous section, let us fix a sequence of positive real numbers $\left(\varepsilon_{j}\right)$ which converges to 0 as $j \rightarrow \infty$. For every $u \in L^{p}(\Omega)$ we define

$$
F^{\prime \prime}(u)=\Gamma-\limsup _{j \rightarrow \infty} F_{\varepsilon_{j}}(u)
$$

In this section we will prove the following proposition, which, together with Corollary 3.2 concludes the proof of Theorem 1.1. 
Proposition 4.1. There exists a constant $\Theta$, with $0<\Theta \leq 60^{\circ}$, such that, if $0<\theta_{0}<\Theta$, then

$$
F^{\prime \prime}(u) \leq \int_{\Omega}|\nabla u(x)|^{2} \mathrm{~d} x+\mu \mathcal{H}^{1}\left(S_{u}\right)
$$

for any $u \in L^{p}(\Omega) \cap G S B V(\Omega)$.

In order to obtain this estimate we need the following lemma, where the hypothesis that $\Omega$ has a Lipschitz boundary plays a crucial role. We use the notation $D^{\alpha} u=D_{1}^{\alpha_{1}} D_{2}^{\alpha_{2}} u=\partial^{\alpha_{1}+\alpha_{2}} u / \partial^{\alpha_{1}} x_{1} \partial^{\alpha_{2}} x_{2}$ for every multiindex $\alpha=\left(\alpha_{1}, \alpha_{2}\right) \in \mathbb{N}^{2}$.

Lemma 4.2. Let $u \in S B V(\Omega) \cap L^{\infty}(\Omega)$, with $\int_{\Omega}|\nabla u|^{2} \mathrm{~d} x+\mathcal{H}^{1}\left(S_{u}\right)<+\infty$, and let $\Omega^{\prime}=(a, b) \times(c, d) \subset \mathbb{R}^{2}$ be an open square such that $\Omega \subset \subset \Omega^{\prime}$. Then $u$ has an extension $v \in S B V\left(\Omega^{\prime}\right) \cap L^{\infty}\left(\Omega^{\prime}\right)$ such that $\int_{\Omega^{\prime}}|\nabla v|^{2} \mathrm{~d} x+$ $\mathcal{H}^{1}\left(S_{v}\right)<+\infty$,

$$
\mathcal{H}^{1}\left(S_{v} \cap \partial \Omega\right)=0
$$

and $\|v\|_{L^{\infty}\left(\Omega^{\prime}\right)}=\|u\|_{L^{\infty}(\Omega)}$. Moreover, there exists a sequence $\left(v_{k}\right)$ in $S B V\left(\Omega^{\prime}\right) \cap L^{\infty}\left(\Omega^{\prime}\right)$ converging to $v$ in $L^{p}\left(\Omega^{\prime}\right)$ as $k \rightarrow \infty$, and for any $k, m_{k}$ disjoint closed segments $L_{1}^{k}, \ldots, L_{m_{k}}^{k} \subset \subset \Omega^{\prime}$, with the following properties:

$$
\begin{gathered}
\left\|v_{k}\right\|_{L^{\infty}\left(\Omega^{\prime}\right)} \leq\|u\|_{L^{\infty}(\Omega)}, \\
\nabla v_{k} \rightarrow \nabla v \text { strongly in } L^{2}\left(\Omega^{\prime} ; \mathbb{R}^{2}\right), \\
\bar{S}_{v_{k}}=\bigcup_{i=1}^{m_{k}} L_{i}^{k} \text { and } \mathcal{H}^{1}\left(\bar{S}_{v_{k}} \backslash S_{v_{k}}\right)=0, \\
\lim _{k \rightarrow \infty} \sum_{i=1}^{m_{k}} \mathcal{H}^{1}\left(L_{i}^{k}\right)=\mathcal{H}^{1}\left(S_{v}\right) .
\end{gathered}
$$

Eventually, we may extend each $v_{k}$ to the whole plane into a compactly-supported function and assume that it is regular, in the sense that $v_{k} \in C^{\infty}\left(\mathbb{R}^{2} \backslash \bigcup_{i=1}^{m_{k}} L_{i}^{k}\right)$, and for any multi-index $\alpha=\left(\alpha_{1}, \alpha_{2}\right) \in \mathbb{N}^{2}$ the partial derivative $D^{\alpha} v_{k}$ is continuous at each end point of the segments $L_{i}^{k}$, while for any (relative) interior point $x \in L_{i}^{k}$ the function $D^{\alpha} v_{k}$ is uniformly continuous in each one of the two connected components of the set $B_{\rho}(x) \backslash L_{i}^{k}$ for $\rho$ is small enough (in particular it admits a finite limit at $x$ from both sides of $L_{i}^{k}$ ).

Proof. We refer to the proof of Theorem 3.9 in [12], which is based on the approximation result obtained in $[16]$.

The regularity we need in the sequel for $v_{k}$ is just $v_{k} \in W^{2, \infty}\left(\mathbb{R}^{2} \backslash \bigcup_{i=1}^{m_{k}} L_{i}^{k}\right)$.

Proof of Proposition 4.1. Since $u \mapsto F^{\prime \prime}(u)$ is lower semi-continuous in $L^{p}(\Omega)$, by (2.1) it is enough to prove (4.1) for a bounded function $u \in L^{\infty}(\Omega) \cap S B V(\Omega)$. Lemma 4.2 provides a function $v \in L^{\infty}\left(\Omega^{\prime}\right) \cap S B V\left(\Omega^{\prime}\right)$ equal to $u$ on $\Omega$, with $\mathcal{H}^{1}\left(S_{v} \cap \partial \Omega\right)=0$. Assume we can construct a sequence $\left(u_{j}\right)$ with $u_{j} \in V_{\varepsilon_{j}}\left(\Omega^{\prime}\right)$ for all $j, u_{j} \rightarrow v$ in $L^{p}\left(\Omega^{\prime}\right)$ as $j \rightarrow \infty$, and

$$
\limsup _{j \rightarrow \infty} \int_{\Omega^{\prime}} \frac{1}{\varepsilon_{j}} f\left(\varepsilon_{j}\left|\nabla u_{j}(x)\right|^{2}\right) \mathrm{d} x \leq \int_{\Omega^{\prime}}|\nabla v(x)|^{2} \mathrm{~d} x+\mu \mathcal{H}^{1}\left(S_{v}\right) .
$$




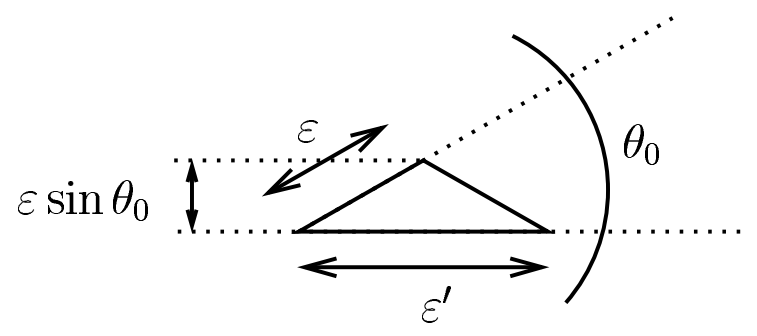

Figure 4.9. The smallest possible triangle $\tilde{T}$ has height $\varepsilon \sin \theta_{0}$. Its longest edge has length $\varepsilon^{\prime}=2 \varepsilon \cos \theta_{0}$.

Using Corollary 3.2 we obtain

$$
\begin{aligned}
\limsup _{j \rightarrow \infty} \int_{\Omega^{\prime}} \frac{1}{\varepsilon_{j}} f\left(\varepsilon_{j}\left|\nabla u_{j}(x)\right|^{2}\right) \mathrm{d} x & \geq \limsup _{j \rightarrow \infty} \int_{\Omega} \frac{1}{\varepsilon_{j}} f\left(\varepsilon_{j}\left|\nabla u_{j}(x)\right|^{2}\right) \mathrm{d} x+\liminf _{j \rightarrow \infty} \int_{\Omega^{\prime} \backslash \bar{\Omega}} \frac{1}{\varepsilon_{j}} f\left(\varepsilon_{j}\left|\nabla u_{j}(x)\right|^{2}\right) \mathrm{d} x \\
& \geq F^{\prime \prime}(u)+\int_{\Omega^{\prime} \backslash \bar{\Omega}}|\nabla v(x)|^{2} \mathrm{~d} x+\mu \mathcal{H}^{1}\left(S_{v} \cap\left(\Omega^{\prime} \backslash \bar{\Omega}\right)\right) .
\end{aligned}
$$

Therefore (4.7) gives

$$
F^{\prime \prime}(u) \leq \int_{\bar{\Omega}}|\nabla v(x)|^{2} \mathrm{~d} x+\mu \mathcal{H}^{1}\left(S_{v} \cap \bar{\Omega}\right),
$$

so that (4.1) holds, since $\Omega$ has a Lipschitz boundary and $\mathcal{H}^{1}\left(S_{v} \cap \partial \Omega\right)=0$.

By a straightforward diagonalization argument, it is enough to construct the functions $u_{j} \in V_{\varepsilon_{j}}\left(\Omega^{\prime}\right)$ when $v$ that has the regularity of the functions $v_{k}$ of Lemma 4.2. We will therefore assume that $v$ is defined in the whole plane, has compact support, and that there exist $m$ disjoint closed segments in $\Omega^{\prime}, L_{1}, \ldots, L_{m}$, such that $\bar{S}_{v}=\bigcup_{i=1}^{m} L_{i}, \mathcal{H}^{1}\left(\bar{S}_{v} \backslash S_{v}\right)=0$, and $v \in W^{2, \infty}\left(\mathbb{R}^{2} \backslash \bigcup_{i=1}^{m} L_{i}\right)$; if $\theta_{0}$ is not too large, we will construct a sequence of functions $u_{j} \in V_{\varepsilon_{j}}\left(\Omega^{\prime}\right)$ such that $u_{j} \rightarrow v$ in $L^{p}\left(\Omega^{\prime}\right)$ and

$$
\limsup _{j \rightarrow \infty} \int_{\Omega^{\prime}} \frac{1}{\varepsilon_{j}} f\left(\varepsilon_{j}\left|\nabla u_{j}(x)\right|^{2}\right) \mathrm{d} x \leq \int_{\Omega^{\prime}}|\nabla v(x)|^{2} \mathrm{~d} x+\mu \sum_{i=1}^{m} \mathcal{H}^{1}\left(L_{i}\right) .
$$

Construction of the sequence $\left(u_{j}\right)$. Let $\varepsilon_{j}^{\prime}=2 \varepsilon_{j} \cos \theta_{0}$,

$$
d=\min \left\{\operatorname{dist}\left(L_{i}, L_{k}\right), \operatorname{dist}\left(L_{i}, \partial \Omega^{\prime}\right): 1 \leq i<k \leq m\right\}>0,
$$

and choose $j$ large enough so that $\varepsilon_{j}^{\prime} \leq d / 10$. We now explain how we construct the triangulation on which $u_{j}$ is defined. In order to simplify the notations we will drop the subscripts $j$ in the sequel, when it is not ambiguous.

Let $\tilde{T}$ be the triangle with minimal height admitted in a triangulation of $\mathcal{T}_{\varepsilon}\left(\Omega^{\prime}\right)$. $\tilde{T}$ is defined up to a translation and a rotation, and is the isosceles triangle shown in Figure 4.9, with two edges of length $\varepsilon$, and two angles equal to $\theta_{0}$. The longest edge of this triangle has length $\varepsilon^{\prime}=2 \varepsilon \cos \theta_{0}$, and the shortest height is $\varepsilon \sin \theta_{0}$.

We first cover each discontinuity set $L_{i}$ with an odd number of triangles $\tilde{T}$, as shown in Figure 4.10. If $N_{i}$ is the integer part of $\mathcal{H}^{1}\left(L_{i}\right) / \varepsilon^{\prime}$, the segment $L_{i}$ can be covered by a strip of width $\varepsilon \sin \theta_{0}$ made of exactly $2 N_{i}+1$ triangles, and the total surface of this strip is estimated by

$$
\left(2 N_{i}+1\right) \frac{\varepsilon^{\prime} \varepsilon \sin \theta_{0}}{2}<\varepsilon \sin \theta_{0}\left(\mathcal{H}^{1}\left(L_{i}\right)+\frac{1}{2} \varepsilon^{\prime}\right) .
$$




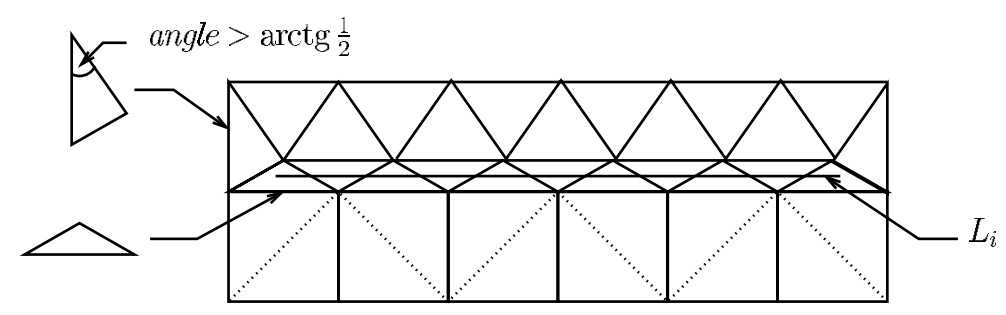

FiguRE 4.10. Each $L_{i}$ is covered with $2 N_{i}+1$ minimal triangles, the whole strip is then included in a larger rectangle $R_{i}$ of size $\left(N_{i}+1\right) \varepsilon^{\prime} \times 2 \varepsilon^{\prime}$.

By adding triangles and squares of side $\varepsilon^{\prime}$ (further divided into triangles by cutting along a diagonal), we can include this strip in a larger rectangle $R_{i}$ (see Fig. 4.10), with a "short" side of length $2 \varepsilon^{\prime}$ and a "long" side of length $\left(N_{i}+1\right) \varepsilon^{\prime}$, and exactly $2 N_{i}+6$ external vertices (or nodes) on $\partial R_{i}$, that we will have to link to some triangulation of $\Omega^{\prime} \backslash R_{i}$.

Provided $\theta_{0} \leq \operatorname{arctg}(1 / 2)$, all the angles of the triangles we have drawn so far are larger than $\theta_{0}$, whereas the length of the edges of these triangles are clearly greater than $\varepsilon$.

We repeat this construction for each $i=1, \ldots, m$. We also denote by $\hat{R}_{i}$ the "enlarged rectangle" $R_{i}$, i.e., the (open) rectangle of size $\left(N_{i}+3\right) \varepsilon^{\prime} \times 4 \varepsilon^{\prime}$ that contains the set $\left\{x \in \Omega^{\prime}: \operatorname{dist}\left(x, R_{i}\right)<\varepsilon^{\prime}\right\}$. Notice that the condition $\varepsilon^{\prime}<d / 10$ ensures that none of the rectangles $\hat{R}_{i}$ intersects another one or the boundary of $\Omega^{\prime}$.

We now consider on $\Omega^{\prime} \backslash \bigcup_{i=1}^{m} \hat{R}_{i}$ a regular triangulation based on the squares $\left[k \varepsilon^{\prime},(k+1) \varepsilon^{\prime}\right] \times\left[l \varepsilon^{\prime},(l+1) \varepsilon^{\prime}\right]$, $k, l \in \mathbb{Z}$, that intersect $\Omega^{\prime}$ but do not touch any of the $\hat{R}_{i}$, each square being then cut into two triangles, along a diagonal. The condition $\varepsilon^{\prime}<d / 10$ ensures that each $\hat{R}_{i}$ is "isolated" in the complement of this triangulation (as it is easy to check that $\operatorname{dist}\left(\hat{R}_{i}, \hat{R}_{k}\right) \geq 3 \varepsilon^{\prime}$ whenever $i \neq k$, and $\operatorname{dist}\left(\hat{R}_{i}, \partial \Omega^{\prime}\right)$ is even larger). We can thus describe independently how the external nodes of each $R_{i}$ have to be connected to the regular "background" triangulation, in order to obtain a global triangulation $\mathbf{T}$ of $\Omega^{\prime}$ that belong to $\mathcal{T}_{\varepsilon}\left(\Omega^{\prime}\right)$. This is done in the Appendix. We describe a particular algorithm that shows how to connect the two triangulations, by drawing new triangles whose edges have length between $\varepsilon^{\prime}$ and $3 \varepsilon^{\prime} \leq 6 \varepsilon$ and whose angles are all greater than $45^{\circ}-\operatorname{arctg}(1 / 2)$. Therefore, provided $\theta_{0} \leq 45^{\circ}-\operatorname{arctg}(1 / 2)$, the global triangulation we construct in this way is in $\mathcal{T}_{\varepsilon}\left(\Omega^{\prime}\right)$.

We define $u_{j}$ as the Lagrange interpolation of $v$ on $T$, i.e., $u_{j}$ is affine in each triangle $T \in \mathbf{T}$, taking the same values as $v$ at the vertices of $T$. A vertex of $T$ might not belong to $\Omega^{\prime}$, but we remind that $v$ is defined in the whole plane. By Theorem 4.4-3 in [24], since the length of the edges of any $T \in \mathbf{T}$ are less than $6 \varepsilon$ and the largest disk contained in $T$ has diameter greater than $2 \varepsilon \cos \theta_{0} \operatorname{tg}\left(\theta_{0} / 2\right)$, there exists a constant $c\left(\theta_{0}\right)$ such that for any such $T$ with $T \cap \bigcup_{i=1}^{m} L_{i}=\emptyset$,

$$
\left\|v-u_{j}\right\|_{H^{1}(T)} \leq c\left(\theta_{0}\right) \varepsilon_{j}\left\|D^{2} v\right\|_{L^{2}\left(T ; \mathbb{R}^{4}\right)},
$$

where $D^{2} v$ is the vector whose components are the second order partial derivatives of $v$. Let $\mathbf{T}_{j}^{0}=\{T \in \mathbf{T}$ : $\left.T \cap \bigcup_{i=1}^{m} L_{i}=\emptyset\right\}$. By (4.9), and since $f \leq f_{\infty}$, we get.

$$
\int_{\Omega^{\prime}} \frac{1}{\varepsilon_{j}} f\left(\varepsilon_{j}\left|\nabla u_{j}(x)\right|^{2}\right) \mathrm{d} x \leq \sum_{T \in \mathbf{T}_{j}^{0}} \frac{\left|T \cap \Omega^{\prime}\right|}{\varepsilon_{j}} f\left(\varepsilon_{j}\left|\nabla u_{j}^{T}\right|^{2}\right)+\mu \sum_{i=1}^{m}\left(\mathcal{H}^{1}\left(L_{i}\right)+\frac{1}{2} \varepsilon_{j}^{\prime}\right)
$$


where $\nabla u_{j}^{T}$ is the (constant) gradient of $u_{j}$ on $T$. Therefore (4.8) will hold if we can show that

$$
\limsup _{j \rightarrow \infty} \sum_{T \in \mathbf{T}_{j}^{0}} \frac{\left|T \cap \Omega^{\prime}\right|}{\varepsilon_{j}} f\left(\varepsilon_{j}\left|\nabla u_{j}^{T}\right|^{2}\right) \leq \int_{\Omega^{\prime}}|\nabla v(x)|^{2} \mathrm{~d} x .
$$

For each $j$ we define the function $U_{j}=\left(\nabla u_{j} \cdot \sum_{T \in \mathbf{T}_{j}^{0}} \chi_{T}\right) \in L^{2}\left(\Omega^{\prime} ; \mathbb{R}^{2}\right)$; we also let $A_{j}=\bigcup_{T \in \mathbf{T}_{j}^{0}} T$. From (4.10) we deduce that there exists some constant $c>0$ such that

$$
\begin{aligned}
\int_{\Omega^{\prime}}\left|\nabla v(x)-U_{j}(x)\right|^{2} \mathrm{~d} x & =\int_{\Omega^{\prime} \cap A_{j}}\left|\nabla v(x)-\nabla u_{j}(x)\right|^{2} \mathrm{~d} x+\int_{\Omega^{\prime} \backslash A_{j}}|\nabla v(x)|^{2} \mathrm{~d} x \\
& \leq c\left(\theta_{0}\right)^{2} \varepsilon_{j}^{2}\left\|D^{2} v\right\|_{L^{2}\left(A_{j} ; \mathbb{R}^{4}\right)}^{2}+\|\nabla v\|_{L^{\infty}\left(\Omega^{\prime} ; \mathbb{R}^{2}\right)}\left|\Omega^{\prime} \backslash A_{j}\right| \\
& \leq c \varepsilon_{j}
\end{aligned}
$$

so that $\left(U_{j}\right)$ tends to $\nabla v$ strongly in $L^{2}\left(\Omega^{\prime} ; \mathbb{R}^{2}\right)$ as $j \rightarrow \infty$. Notice, moreover, that the sequence $\left(U_{j}\right)$ is uniformly bounded in $L^{\infty}\left(\Omega^{\prime} ; \mathbb{R}^{2}\right)$. Indeed, $u_{j}$ being a Lagrange interpolation of $v$, the slope of $u_{j}$ along the edge of any triangle $T \in \mathbf{T}_{j}^{0}$ is less than $\|\nabla v\|_{L^{\infty}\left(\mathbb{R}^{2} ; \mathbb{R}^{2}\right)}$, thus by Remark 3.5

$$
\left\|U_{j}\right\|_{L^{\infty}\left(\Omega^{\prime} ; \mathbb{R}^{2}\right)} \leq\|\nabla v\|_{L^{\infty}\left(\mathbb{R}^{2} ; \mathbb{R}^{2}\right)} \frac{\sqrt{5}}{\sin \theta_{0}}=k<+\infty .
$$

For any $j$ let

$$
\delta_{j}=\sup _{0<t \leq \varepsilon_{j} k^{2}} \frac{f(t)}{t} \geq 1
$$

From the properties of $f$ we know that $\delta_{j} \rightarrow 1$ as $j \rightarrow \infty$. Now,

$$
\begin{aligned}
\sum_{T \in \mathbf{T}_{j}^{0}} \frac{\left|T \cap \Omega^{\prime}\right|}{\varepsilon_{j}} f\left(\varepsilon_{j}\left|\nabla u_{j}^{T}\right|^{2}\right) & =\int_{\Omega^{\prime}} \frac{1}{\varepsilon_{j}} f\left(\varepsilon_{j}\left|U_{j}(x)\right|^{2}\right) \mathrm{d} x \\
& \leq \int_{\Omega^{\prime}} \frac{1}{\varepsilon_{j}} \delta_{j} \varepsilon_{j}\left|U_{j}(x)\right|^{2} \mathrm{~d} x=\delta_{j} \int_{\Omega^{\prime}}\left|U_{j}(x)\right|^{2} \mathrm{~d} x
\end{aligned}
$$

hence (4.11) holds. This achieves the proof of (4.8), so that (4.1) holds true. From this inequality and from Corollary 3.2 we deduce that $\left(F_{\varepsilon}\right) \Gamma$-converges to $F$ in $L^{p}(\Omega)$ as $\varepsilon \rightarrow 0$ for $0<\theta_{0} \leq 45^{\circ}-\operatorname{arctg}(1 / 2)$, so that Theorem 1.1 is proved.

\section{Proof of the CONVERGence Result}

Proof of Theorem 1.2. Let $g \in L^{\infty}(\Omega)$ and for each $\varepsilon>0$, let $u_{\varepsilon} \in V_{\varepsilon}(\Omega)$ be a minimizer of

$$
F_{\varepsilon}(u)+\int_{\Omega}|u(x)-g(x)|^{p} \mathrm{~d} x .
$$


Notice that $\sup _{\varepsilon>0}\left\{F_{\varepsilon}\left(u_{\varepsilon}\right)+\int_{\Omega}\left|u_{\varepsilon}(x)-g(x)\right|^{p} \mathrm{~d} x\right\} \leq c=\int_{\Omega}|g(x)|^{p} \mathrm{~d} x<+\infty$. Choose some $\delta>0$ and construct, for all $\varepsilon>0, v_{\varepsilon}$ as in Proposition 3.3 with $A=\Omega$. As

$$
(1-\delta) \int_{\Omega_{\omega(\varepsilon)}}\left|\nabla v_{\varepsilon}(x)\right|^{2} \mathrm{~d} x+c_{\delta} \mathcal{H}^{1}\left(S_{v_{\varepsilon}} \cap \Omega_{\omega(\varepsilon)}\right) \leq c
$$

and

$$
\left\|v_{\varepsilon}\right\|_{L^{p}(\Omega)} \leq\left\|u_{\varepsilon}\right\|_{L^{p}(\Omega)} \leq 2 c^{1 / p},
$$

we deduce from Ambrosio's Theorem 2.1 that for any $\eta>0$, there exists a subsequence $\left(v_{\varepsilon_{j}}\right)$ and $u \in G S B V\left(\Omega_{\eta}\right)$ such that $v_{\varepsilon_{j}}(x)$ tends to $u(x)$ a.e. in $\Omega_{\eta}$.

Using a diagonal procedure we may therefore construct a subsequence of $\left(v_{\varepsilon}\right)$, denoted by $\left(v_{j}\right)$ (with $v_{j}=v_{\varepsilon_{j}}$, and we will denote as well the functions $u_{\varepsilon_{j}}$ by $\left.u_{j}\right)$, and $u \in G S B V(\Omega)$, such that $v_{j}(x)$ tends to $u(x)$ a.e. in $\Omega$. By (5.1) and Fatou's lemma $u \in L^{p}(\Omega)$, and for any $\rho>0$, if $j$ is large enough,

$$
\begin{aligned}
\int_{\Omega}\left|v_{j}(x)-u(x)\right| \mathrm{d} x & \leq \rho|\Omega|+\int_{\Omega}\left|v_{j}(x)-u(x)\right| \chi_{\left\{\left|v_{j}-u\right|>\rho\right\}}(x) \mathrm{d} x \\
& \leq \rho|\Omega|+\left\|v_{j}-u\right\|_{L^{p}(\Omega)}\left|\left\{\left|v_{j}-u\right|>\rho\right\}\right|^{1 / p^{\prime}} \\
& \leq \rho(|\Omega|+1)
\end{aligned}
$$

where $p^{\prime}=p /(p-1)$ (recall that $p>1$ ). Thus $\left(v_{j}\right)$ converges to $u$ strongly in $L^{1}(\Omega)$, and the same kind of argument, together with (3.6), shows that $\left(u_{j}\right)$ also converges strongly to $u$ in $L^{1}(\Omega)$ as $j$ goes to infinity. Invoking the $\Gamma$-convergence result of Theorem 1.1 in the strong topology of $L^{1}(\Omega)$ we deduce that

$$
F(u) \leq \liminf _{j \rightarrow \infty} F_{\varepsilon_{j}}\left(u_{j}\right) .
$$

Obviously, $u$ is also the weak limit in $L^{p}(\Omega)$ of $u_{j}$, therefore

$$
\int_{\Omega}|u(x)-g(x)|^{p} \mathrm{~d} x \leq \liminf _{j \rightarrow \infty} \int_{\Omega}\left|u_{j}(x)-g(x)\right|^{p} \mathrm{~d} x
$$

and combining the last two inequalities we see that

$$
F(u)+\int_{\Omega}|u(x)-g(x)|^{p} \mathrm{~d} x \leq \liminf _{j \rightarrow \infty}\left\{F_{\varepsilon_{j}}\left(u_{j}\right)+\int_{\Omega}\left|u_{j}(x)-g(x)\right|^{p} \mathrm{~d} x\right\} .
$$

Now, let $u^{\prime} \in G S B V(\Omega) \cap L^{p}(\Omega)$. We proved in Section 4 that there exists a sequence $\left(u_{j}^{\prime}\right)$ converging to $u$ in $L^{p}(\Omega)$ such that

$$
\limsup _{j \rightarrow \infty} F_{\varepsilon_{j}}\left(u_{j}^{\prime}\right) \leq F\left(u^{\prime}\right)
$$

As

$$
F_{\varepsilon_{j}}\left(u_{j}\right)+\int_{\Omega}\left|u_{j}(x)-g(x)\right|^{p} \mathrm{~d} x \leq F_{\varepsilon_{j}}\left(u_{j}^{\prime}\right)+\int_{\Omega}\left|u_{j}^{\prime}(x)-g(x)\right|^{p} \mathrm{~d} x,
$$

we deduce from $(5.4,5.5)$ that

$$
F(u)+\int_{\Omega}|u(x)-g(x)|^{p} \mathrm{~d} x \leq F\left(u^{\prime}\right)+\int_{\Omega}\left|u^{\prime}(x)-g(x)\right|^{p} \mathrm{~d} x,
$$


showing the minimality of $u$. Moreover, if we choose $u^{\prime}=u$, inequalities $(5.4,5.5,5.6)$ also yield

$$
\lim _{j \rightarrow \infty}\left\{F_{\varepsilon_{j}}\left(u_{j}\right)+\int_{\Omega}\left|u_{j}(x)-g(x)\right|^{p} \mathrm{~d} x\right\}=F(u)+\int_{\Omega}|u(x)-g(x)|^{p} \mathrm{~d} x,
$$

which, together with $(5.2,5.3)$, shows that $\left\|u_{j}-g\right\|_{L^{p}(\Omega)}$ tends to $\|u-g\|_{L^{p}(\Omega)}$. As $p>1$, we conclude that $\left(u_{j}\right)$ converges to $u$ strongly in $L^{p}(\Omega)$, and this achieves the proof of Theorem 1.2.

The assertion in Remark 1.4 comes from the fact that, by the maximum principle, any minimizer of the Mumford and Shah problem

$$
\min _{u \in L^{p}(\Omega)}\left\{F(u)+\int_{\Omega}|u(x)-g(x)|^{p} \mathrm{~d} x\right\}
$$

must satisfy $|u(x)| \leq\|g\|_{L^{\infty}(\Omega)}$ a.e. in $\Omega$, so that is is enough to consider in the above proof functions $u$ that satisfy this constraint. We conclude by noticing that the sequence constructed in Section 4 also satisfies $\left\|u_{j}\right\|_{L^{\infty}(\Omega)} \leq\|u\|_{L^{\infty}(\Omega)}$ for all $j$.

Part of this work was done while the first author was visiting SISSA in the autumn of 1996. This work is part of the project INTAS-96-1061 "Homogenization of Problems of Mathematical Physics" and of the project "Relaxation and Homogenization Methods in the Study of Composite Materials", Progetto Strategico CNR, 1995, "Matematica per la Tecnologia e la Società".

\section{APPENDIX}

In this section we show how to construct the triangulation needed in the proof of (4.8). It is not restrictive to assume that $\varepsilon^{\prime}=1$, and to consider the following "renormalized" situation. Fix $m \in \mathbb{N}, m \geq 3$, and let $R=[0,2] \times[0, m]$ and $\hat{R}=]-1,3[\times]-1, m+1\left[\right.$. Let $\alpha \in\left[-45^{\circ}, 45^{\circ}\right]$ be an angle, $u_{\alpha}=(\cos \alpha, \sin \alpha)$ and $v_{\alpha}=(-\sin \alpha, \cos \alpha), \xi^{0} \in\left[0,1\left[\times\left[0,1\left[\right.\right.\right.\right.$ and for all $(k, l) \in \mathbb{Z}^{2}, \xi_{k, l}=\xi^{0}+k u_{\alpha}+l v_{\alpha}$. Consider now a "background" triangulation $\mathbf{B}$ in $\mathbb{R}^{2}$ based on the closed squares $\left[\xi_{k, l}, \xi_{k+1, l}, \xi_{k+1, l+1}, \xi_{k, l+1}\right]=C_{k, l}$ that do not touch $\hat{R}$, each of those being then cut along a diagonal. In order to construct a global triangulation $\mathbf{T}$ (here, of $\mathbb{R}^{2}$ ) containing the triangles of $R$ and $\mathbf{B}$, we need to connect the $2 m+4$ external nodes of $R$, namely, $(0, k),(2, k), k=0, \ldots, m$, $(1,0)$ and $(1, m)$, to the triangulation $\mathbf{B}$, that has a different orientation (see Fig. 5.11).

First notice that, by symmetry, it is not restrictive to assume that $0 \leq \alpha \leq 45^{\circ}$. Let $S$ be the union of all the $C_{k, l},(k, l) \in \mathbb{Z}^{2}$, whose vertices are all in $\mathbb{R}^{2} \backslash \hat{R}$. The set $S$ covers the whole triangulation $\mathbf{B}$, and might also contain (at most) four additional squares, at each corner of the rectangle $\hat{R}$ (since at the corner there might be a square $C_{k, l}$ with an edge that intersects $\hat{R}$ but all four vertices outside of $\hat{R}$ ). We put inside our global triangulation $\mathbf{T}$ the triangles obtained by cutting along a diagonal each one of these four possible squares.

Let $S^{\prime}$ be the complement of $S$, or more precisely the union of the squares $C_{k, l}$ that have at least one vertex in $\hat{R}$. It is not difficult to check that $\partial S=\partial S^{\prime}=S \cap S^{\prime}$.

An easy study shows that the distance from any square of $S$ to $R$ is at least $\sqrt{2}-(1 / 2)$, so that

$$
S \cap\left\{x \in \mathbb{R}^{2}: \operatorname{dist}(x, R)<\sqrt{2}-\frac{1}{2}\right\}=\emptyset
$$

thus

$$
\left\{x \in \mathbb{R}^{2}: \operatorname{dist}(x, R)<\sqrt{2}-\frac{1}{2}\right\} \subset S^{\prime}
$$

Moreover, if $C_{k, l} \subset S^{\prime}$, one vertex of $C_{k, l}$ (say, $\xi_{i, j}$, with $i \in\{k, k+1\}$ and $j \in\{l, l+1\}$ ) lies inside $\hat{R}$, so that the four squares $C_{i, j}, C_{i-1, j}, C_{i, j-1}$, and $C_{i-1, j-1}$ are also contained in $S^{\prime}$. In particular, the disc of center $\xi_{i, l}$ and radius 1 is in $S^{\prime}$, and at least one point on the boundary of this disc is at distance less than $\sqrt{2}-1$ from $R$, 


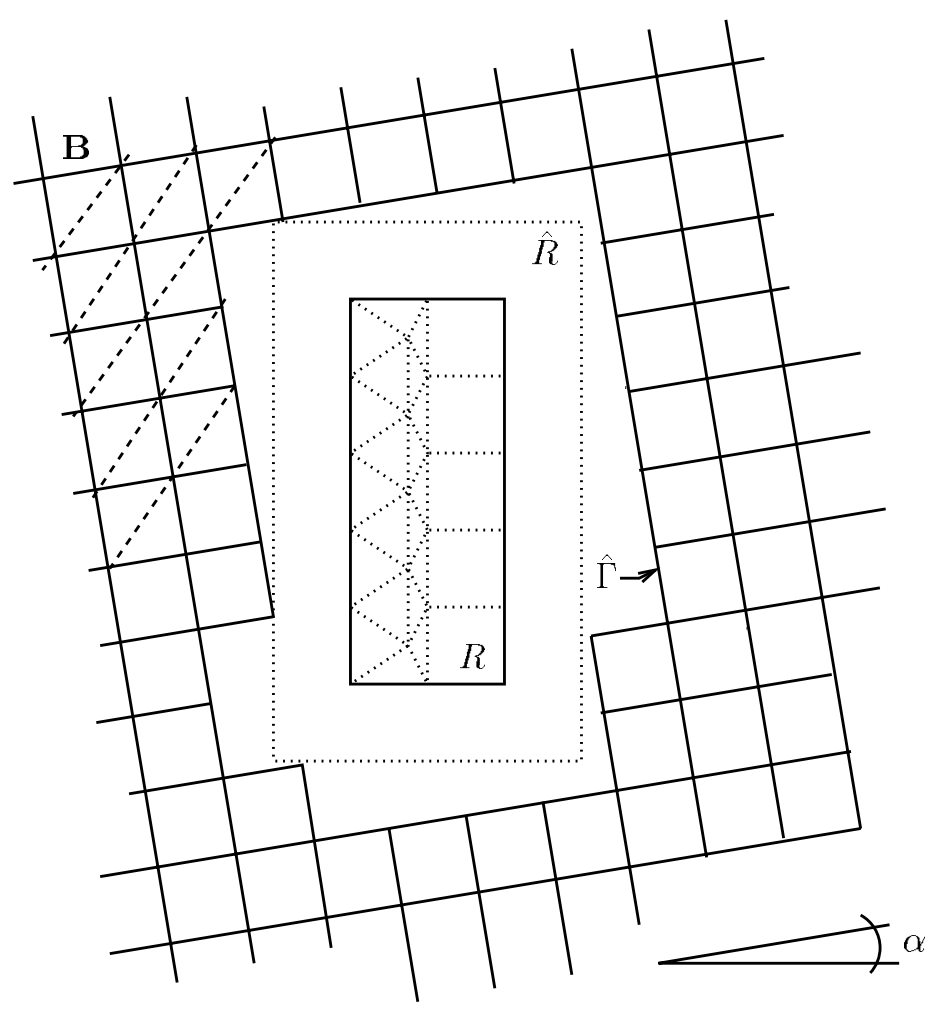

FiguRE 5.11. The two triangulations that have to be connected.

so that we can find in $S^{\prime}$ a path from any point of $C_{k, l}$ to some point of $\left\{x \in \mathbb{R}^{2}: \operatorname{dist}(x, R)<\sqrt{2}-1 / 2\right\}$. Together with (A.1) it shows that $S^{\prime}$ is connected.

To show that $S$ is connected we use the following argument. Notice first that for any $x \notin \hat{R}$, the rectangle $\hat{R}$ lies entirely on one side of either the horizontal or the vertical line going through $x$. If $C_{k, l}$ is a square in $S$, we can draw through each vertex the horizontal or vertical line having this property. Assume that for each vertex $C_{k, l}$ lies on the same side of the corresponding line as $\hat{R}$. Then, the four lines delimit a square that contains $\hat{R}$, but since the diameter of this square is at most 2 , this is impossible. Therefore, one of those four lines must cut through $C_{k, l}$, so that two consecutive vertices of $C_{k, l}$ lie on the side opposite to $\hat{R}$. Assume those the line through $\xi_{k, l}$ is vertical, and that $\hat{R}$ lies on the left of this line, while $\xi_{k+1, l}$ lies on the right; then, all the squares $C_{k, l^{\prime}}$ for $l^{\prime} \geq l$ lie in $S$, so that each $x \in C_{k, l}$ is connected to infinity through some half-line in $\bigcup_{l^{\prime}>l} C_{k, l^{\prime}} \subset S$. The other cases can be treated in a similar way, so that each point of $S$ is connected to infinity through a half line contained in $S$. Since $S^{\prime}$ is bounded, this shows that $S$ is connected and that $S^{\prime}$ is simply connected.

Invoking Jordan's lemma we deduce that $\hat{\Gamma}=\partial S=\partial S^{\prime}=S \cap S^{\prime}$ is a Jordan curve. It is clearly made of segments $\left[\xi_{k, l}, \xi_{k+1, l}\right]$ or $\left[\xi_{k, l}, \xi_{k, l+1}\right]$, and since

$$
S^{\prime} \subset\left\{x \in \mathbb{R}^{2}: \operatorname{dist}(x, \hat{R}) \leq \sqrt{2}\right\},
$$

we deduce that this inclusion also holds for $\hat{\Gamma}$.

Now, we slightly modify the curve $\hat{\Gamma}$, in the following way. Each time $\hat{\Gamma}$ goes along two consecutive edges of some square $C_{k, l} \subset S^{\prime}$, say, $[x, y]$ and then $[y, z]$, we replace these two edges by the diagonal $[x, z]$, and we 


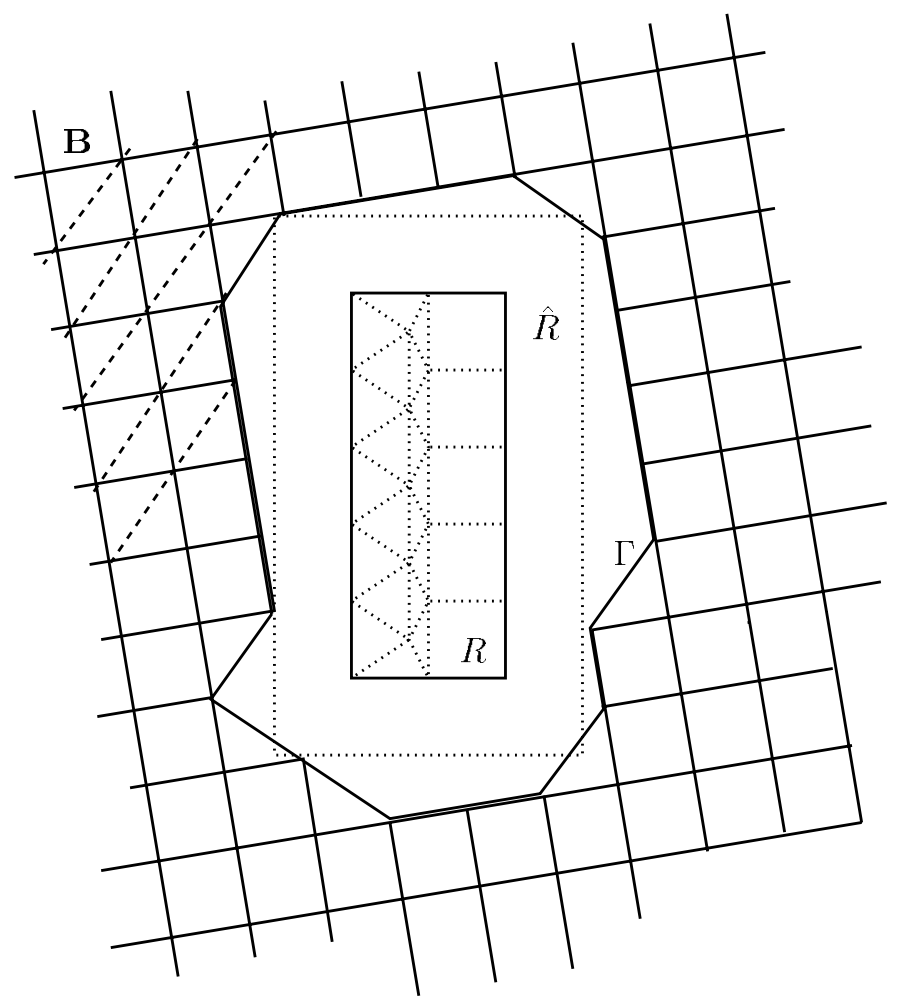

Figure 5.12. The curve $\Gamma$.

put the triangle $[x, y, z]$ in our global triangulation $\mathbf{T}$. We call $\Gamma$ the new curve obtained in this way; it is not difficult to check that $\Gamma \subset[-2,4] \times[-2, m+2]$ (since each vertex of $\hat{\Gamma}$ that was lying out of this rectangle has been "eliminated" by the above described procedure). The new curve $\Gamma$ is made of segments $[x, y]$ with $\pm(y-x)=v_{\alpha}$ or $u_{\alpha}+v_{\alpha}$ along the vertical sides of $R$, and $\pm(y-x)=u_{\alpha}$ or $u_{\alpha}-v_{\alpha}$ along the two small horizontal sides of $R$.

We need now to explain how we connect the nodes of $R$ to the nodes of $\Gamma$ (i.e., the points $\xi_{k, l}$ that lie on the curve $\Gamma$ ). We will first show how to connect every node in $\{(0, k): 0 \leq k \leq m\}$ to the nodes of $\Gamma \cap[-2,-1] \times[-1, m+1[$. Then, the nodes of $R$ in $\{(0,0),(1,0),(2,0)\}$ will be connected to the nodes of $\Gamma \cap]-1,3] \times[-2,-1]$ in the same way, just by replacing "up" and "down" with "left" and "right" in the following explanations, whereas exchanging "up" and "down", the reader will understand how to connect the nodes of $R$ in $\{(2, k): 0 \leq k \leq m\}$ to the nodes of $\Gamma \cap[3,4] \times]-1, m+1]$, and replacing "up" and "down" with "right" and "left" he will get the algorithm to connect the nodes in $\{(0, m),(1, m),(2, m)\}$ to the nodes of $\Gamma \cap[-1,3[\times[m+1, m+2]$. Eventually, we will describe how we construct the triangles at each corner of $R$.

Let $\Gamma^{0}$ be the set $\left\{\xi_{k, l}: k, l \in \mathbb{Z}\right\} \cap \Gamma \cap[-2,-1] \times[-1, m+1[$ of the nodes of the vertical part of $\Gamma$ along the left edge of $R$. A node $n=(0, k)(0 \leq k \leq m)$ is connected to a node in $\Gamma^{0}$ according to the three following rules:

(a) $n$ is connected to the lowest node in $[-2,-1] \times[k, k+1[$ (i.e., above $n$ but below $(0, k+1)$, see Fig. 5.13);

(b) $n$ is connected to all the nodes in $[-2,-1] \times[k-1, k[$;

(c) $n$ is connected to the highest node in $[-2,-1] \times[-1, k[$. 

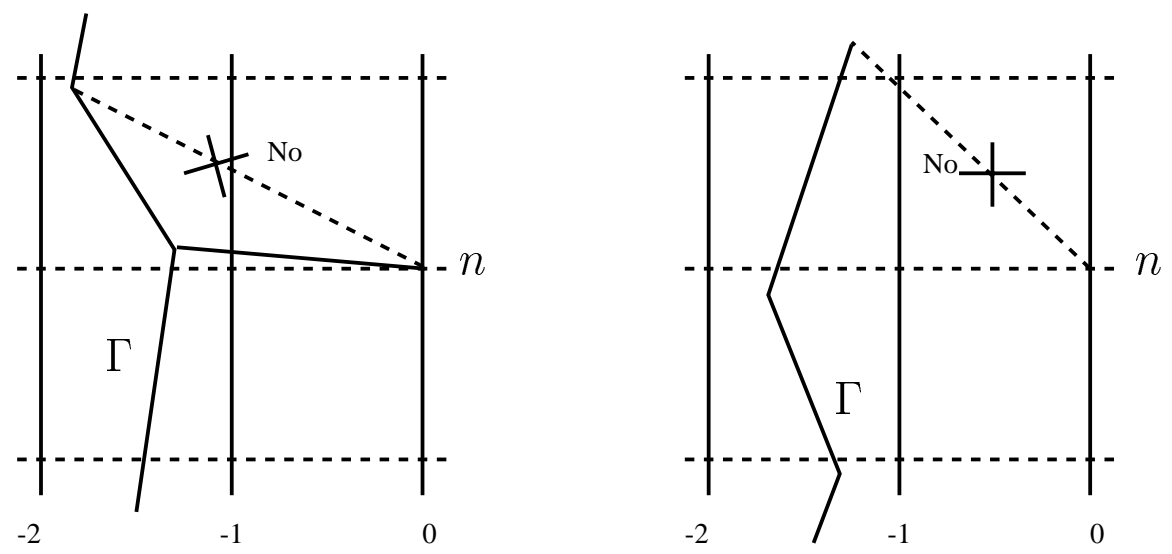

Figure 5.13. Rule (a).
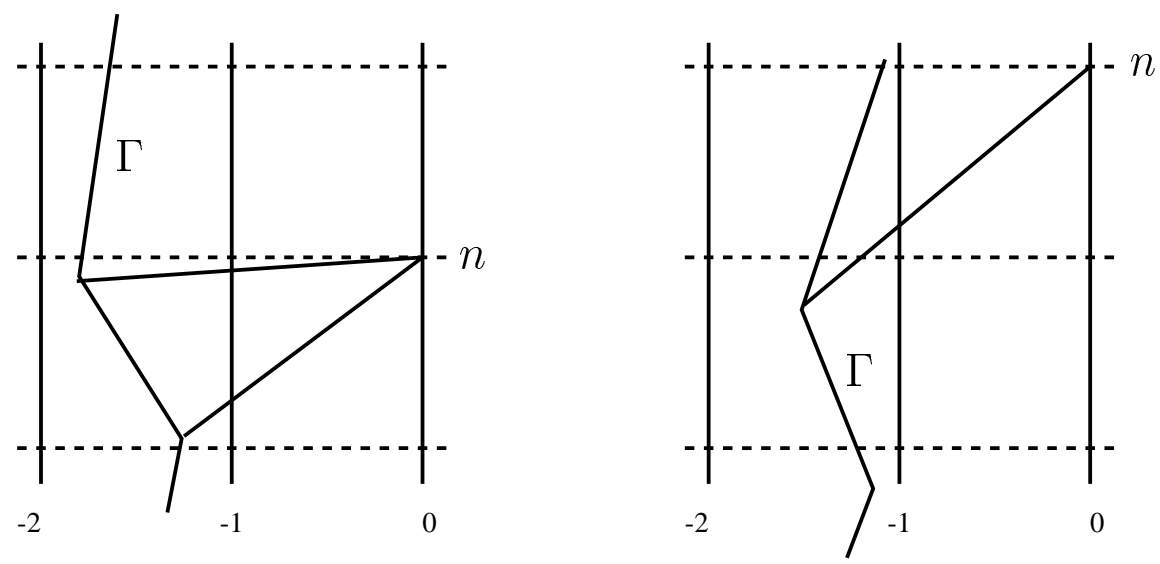

Figure 5.14. Rule (b) (left) and (c) (right).

Rule (c) is useless if $\Gamma^{0} \cap[-2,-1] \times[k-1, k[$ is not empty, as in this case the connection is already ensured according to rule (b) (see Fig. 5.14).

Notice that, according to rule (a), $n$ may remain unconnected to all the nodes above, if $\Gamma^{0} \cap[-2,-1] \times[k, k+1[$ is empty. This is necessary to ensure that none of the segments we draw intersects some other.

It is not difficult to check that, with this method, the whole strip between the left edge of $R$ and $\Gamma$ is covered up with triangles. In particular, we check that there is at most one node of $\Gamma^{0}$ in the square $[-2,-1] \times[m, m+1[$ (as if $x$ were the highest node in this square and if $x-v_{\alpha} \in[-2,-1] \times\left[m, m+1\left[\right.\right.$, then $\left(x+u_{\alpha}\right)_{2} \geq m+1$ so that the curve $\Gamma$ should directly connect $x-v_{\alpha}$ to $x+u_{\alpha}$, and $x$ could not be on $\Gamma$ ), so that the highest node of $\Gamma^{0}$ has to be linked to $(0, m)$ according to rule (a) or (b).

A more accurate study shows that the triangles we have constructed are of five different kinds; then, studying every type of triangle, we may establish that their angles are all greater than $\hat{\theta}=45^{\circ}-\operatorname{arctg}(1 / 2)>18^{\circ}$.

We now explain how we deal with the corners. If $x$ is the lowest node of the curve of $\Gamma \cap[-2,-1] \times[-1, m+1[$ and $y$ the last node on the left of $\Gamma \cap]-1,3] \times[-2,-1]$, we know that the above described algorithm connects both points $x$ and $y$ to $(0,0)$. We simply join $x$ to $y$, if $[x, y]$ is not already part of $\Gamma$. Indeed, we may check that in this case there is at most one point $z \in[-2,-1] \times[-2,-1]$ such that $\Gamma$ goes from $x$ to $y$ along $[x, z]$ 


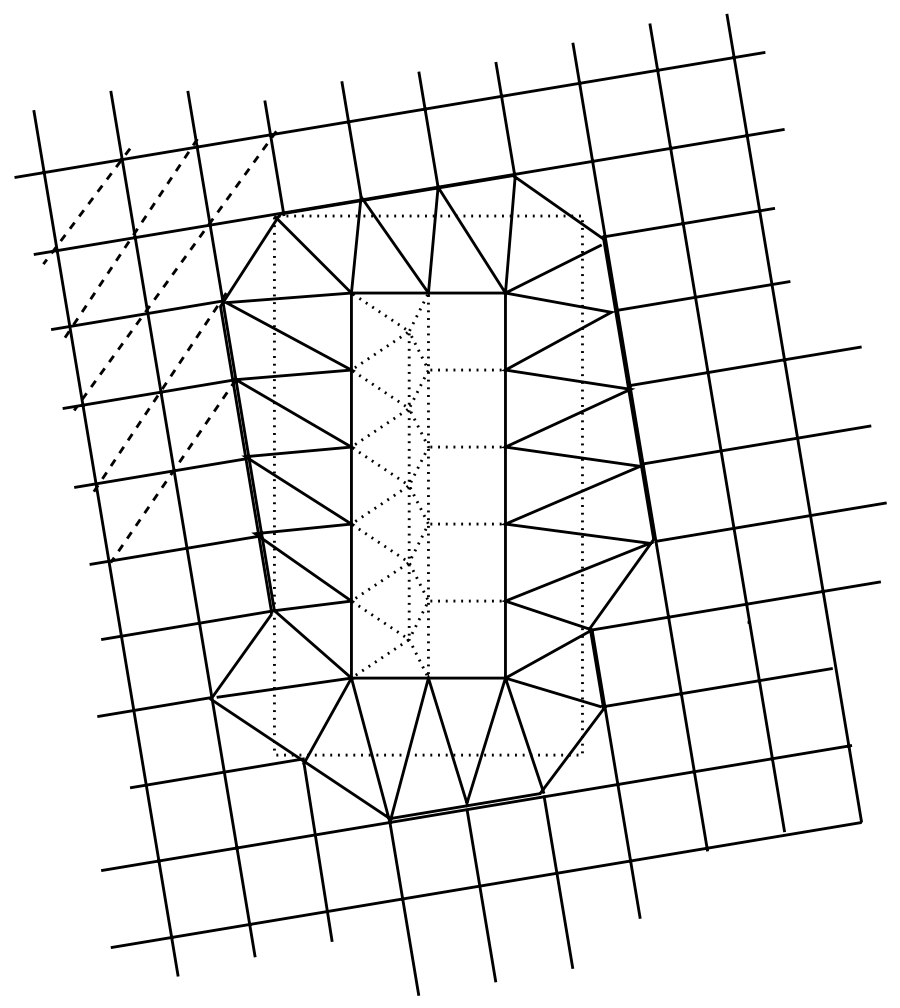

Figure 5.15. The triangulation $\mathbf{T}$.

and $[z, y]$, so that we have created two triangles $[x, y, z]$ and $[x, y,(0,0)]$. Once again, we check that the angles of the triangles constructed in this way are greater than $\hat{\theta}$. Notice eventually that the longest edge created by the whole algorithm has length less than $\sqrt{8}<3$. Figure 5.15 shows the final triangulation $\mathbf{T}$.

\section{REFERENCES}

[1] L. Ambrosio, A compactness theorem for a new class of functions of bounded variation. Boll. Un. Mat. Ital. B 3 (1989) $857-881$.

[2] L. Ambrosio, Variational problems in $S B V$ and image segmentation. Acta Appl. Math. 17 (1989) 1-40.

[3] L. Ambrosio, Existence theory for a new class of variational problems. Arch. Rational Mech. Anal. 111 (1990) $291-322$.

[4] L. Ambrosio and V.M. Tortorelli, Approximation of functionals depending on jumps by elliptic functionals via $\Gamma$-convergence. Comm. Pure Appl. Math. 43 (1990) 999-1036.

[5] L. Ambrosio and V.M. Tortorelli, On the approximation of free discontinuity problems. Boll. Un. Mat. Ital. B 6 (1992) $105-123$.

[6] H. Attouch, Variational Convergence for Functions and Operators. Pitman, London (1984).

[7] G. Bellettini and A. Coscia, Discrete approximation of a free discontinuity problem. Numer. Funct. Anal. Optim. 15 (1994) 201-224.

[8] G. Bouchitté, A. Braides and G. Buttazzo, Relaxation results for some free discontinuity problems. J. Reine Angew. Math. 458 (1995) 1-18.

[9] B. Bourdin and A. Chambolle, Implementation of an adaptive finite-elements approximation of the Mumford-Shah functional. Preprint LPMTM, Université Paris-Nord/CEREMADE, Université de Paris-Dauphine (1998); Numer. Math. (to appear).

[10] A. Braides and G. Dal Maso, Non-Local Approximation of the Mumford-Shah Functional. Calc. Var. Partial Differential Equations 5 (1997) 293-322. 
[11] A. Chambolle, Image segmentation by variational methods: Mumford and Shah functional and the discrete approximations. SIAM J. Appl. Math. 55 (1995) 827-863.

[12] G. Cortesani, Strong approximation of $G S B V$ functions by piecewise smooth functions. Ann. Univ. Ferrara Sez. VII (N.S.) (to appear).

[13] G. Dal Maso, An Introduction to $\Gamma$-Convergence. Birkhäuser, Boston (1993).

[14] E. De Giorgi and L. Ambrosio, Un nuovo funzionale del calcolo delle variazioni. Atti Accad. Naz. Lincei Rend. Cl. Sci. Fis. Mat. Natur. 82 (1988) 199-210.

[15] E. De Giorgi and T. Franzoni, Su un tipo di convergenza variazionale. Atti Accad. Naz. Lincei Rend. Cl. Sci. Fis. Mat. Natur. 58 (1975) 842-850.

[16] F. Dibos and E. Séré, An approximation result for the minimizers of Mumford-Shah functional. Boll. Un. Mat. Ital. A 11 (1997) 149-162.

[17] L.C. Evans and R.F. Gariepy, Measure Theory and Fine Properties of Functions. CRC Press, Boca Raton (1992).

[18] H. Federer, Geometric Measure Theory. Springer-Verlag, New York (1969).

[19] J. Frehse, Capacity methods in the theory of partial differential equations. Jahresber. Deutsch. Math.-Verein. 84 (1982) 1-44.

[20] E. Giusti, Minimal Surfaces and Functions of Bounded Variation. Birkhäuser, Basel (1983).

[21] J.M. Morel and S. Solimini, Variational Models in Image Segmentation. Birkhäuser, Boston, (1995).

[22] D. Mumford and J. Shah, Boundary detection by minimizing functionals, I, in Proc. IEEE Conf. on Computer Vision and Pattern Recognition, San Francisco (1985).

[23] D. Mumford and J. Shah, Optimal approximation by piecewise smooth functions and associated variational problems. Comm. Pure Appl. Math. 42 (1989) 577-685.

[24] P.A. Raviard and J.M. Thomas, Introduction à l'analyse numérique des équations aux dérivées partielles. Masson, Paris (1983).

[25] W.P. Ziemer, Weakly Differentiable Functions. Springer-Verlag, Berlin (1989). 